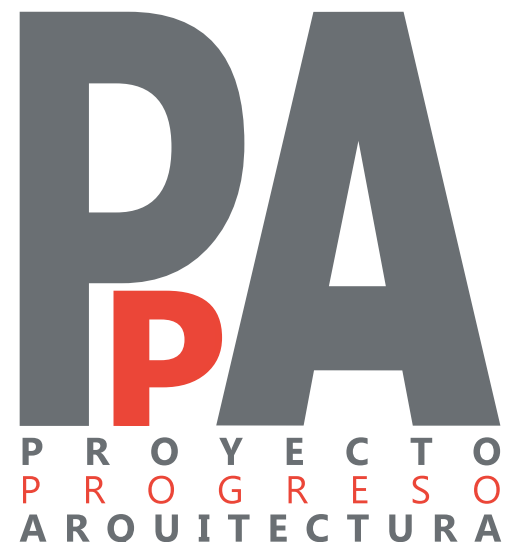

ARQUITECTURAS AMPLIADAS

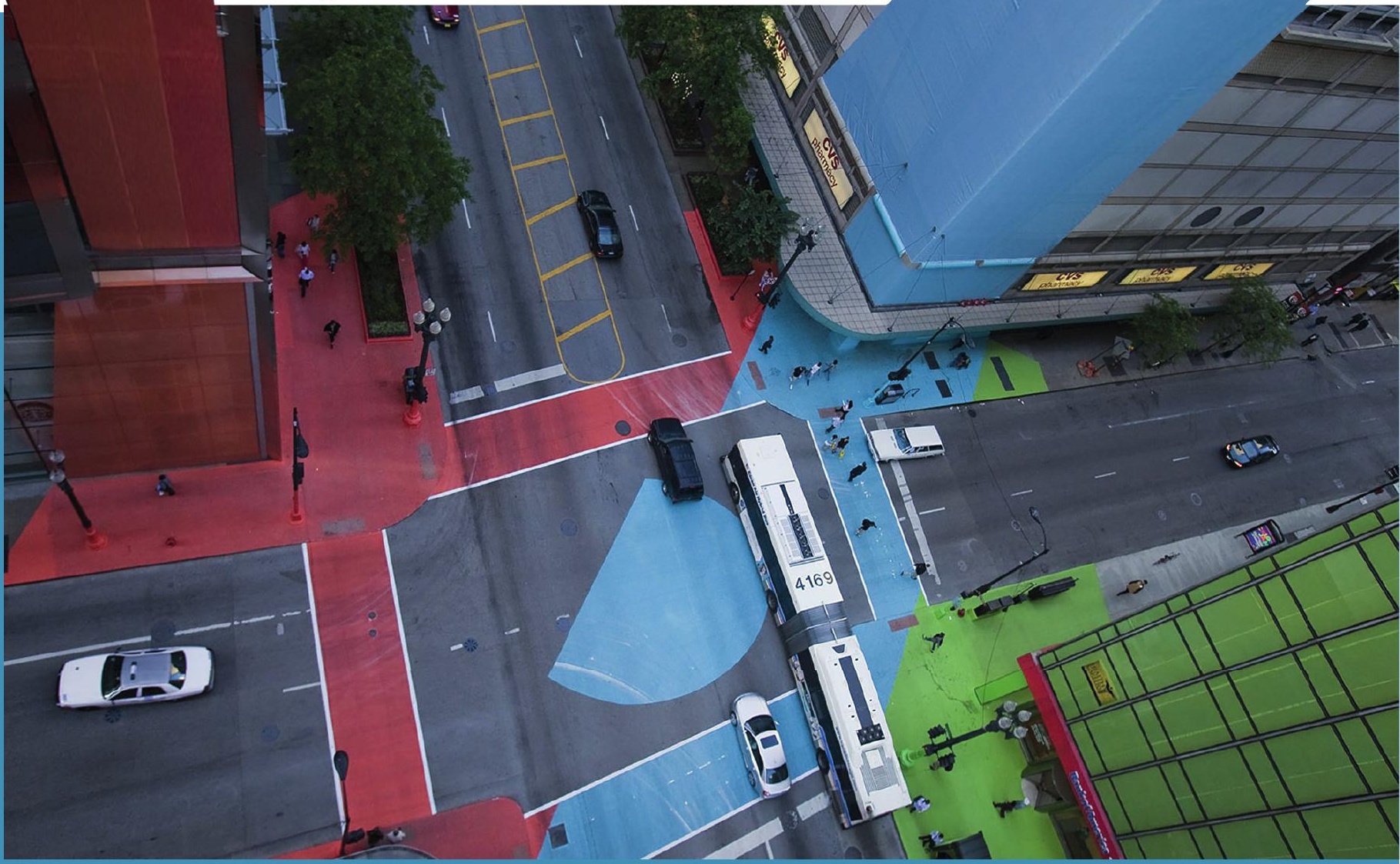




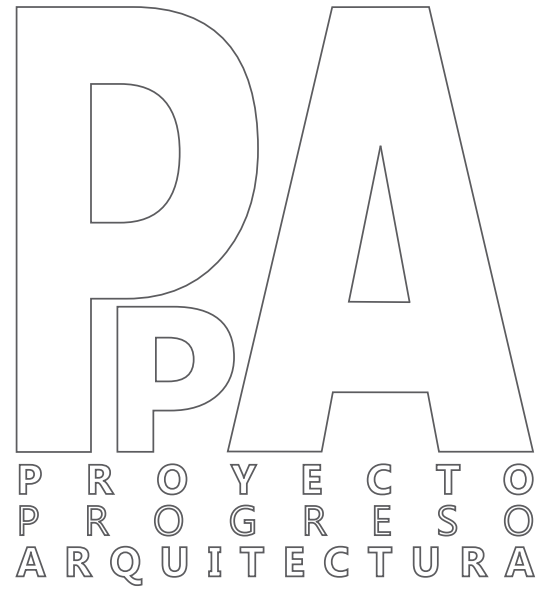

\section{ARQUITECTURAS AMPLIADA}

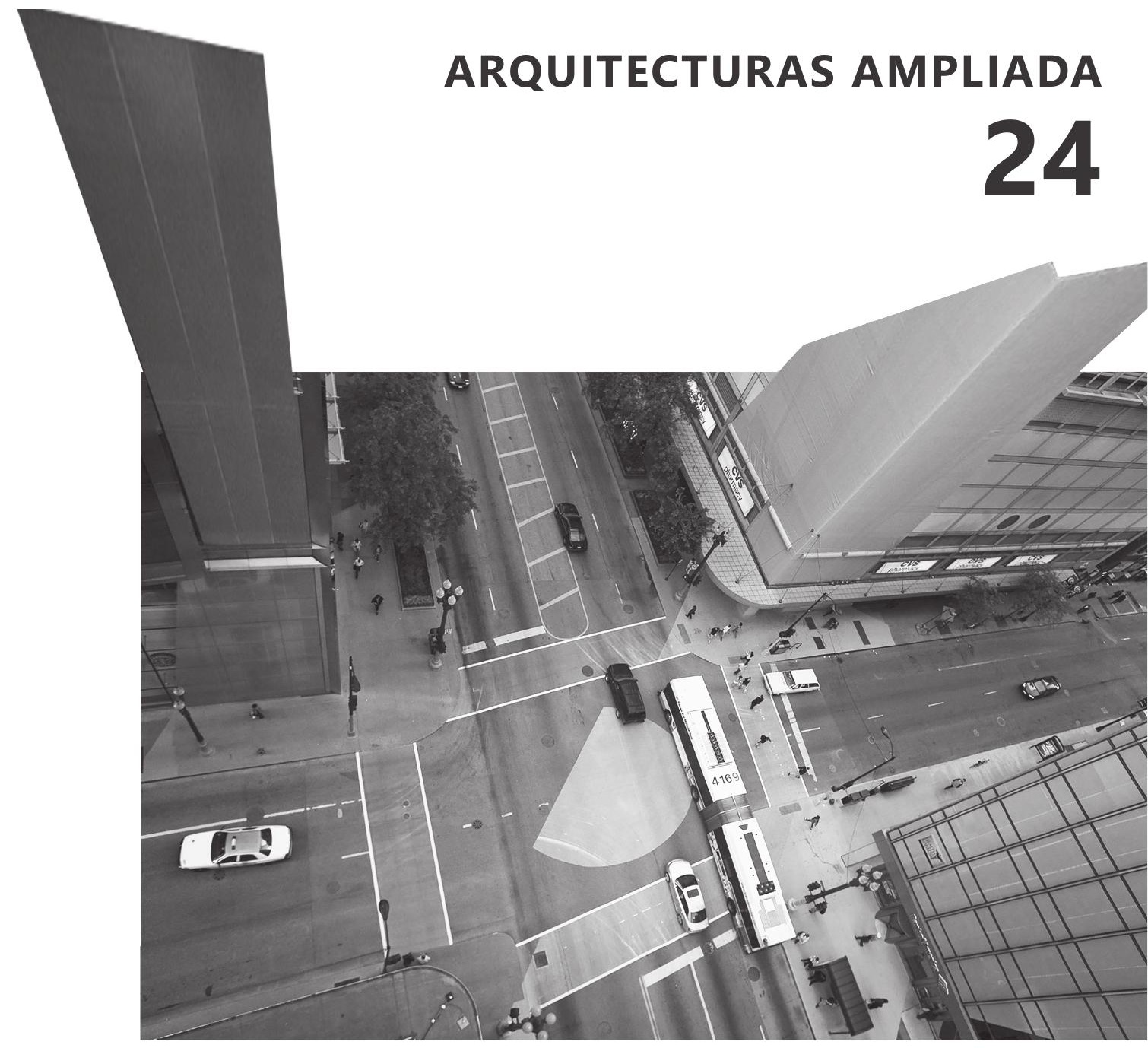

EDITORIAL UNIVERSIDAD DE SEVILLA AÑO 2019. ISSN 2171-6897 ISSNe 2173-1616 DOI: http://dx.doi.org/10.12795/ppa 


\section{REVISTA PROYECTO PROGRESO ARQUITECTURA}

N2/4

arquitecturas ampliadas
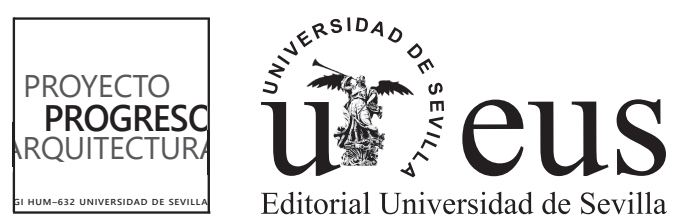


\section{arquitecturas ampliadas}

EDITA

Editorial Universidad de Sevilla. Sevilla

DIRECCIÓN CORRESPONDENCIA CIENTÍFICA

E.T.S. de Arquitectura. Avda Reina Mercedes, nº $241012-$

Sevilla.

Amadeo Ramos Carranza, Dpto. Proyectos Arquitectónicos.

e-mail: revistappa.direccion@gmail.com

\section{EDICIÓN ON-LINE}

Portal informático https://revistascientificas.us.es/index.php/ppa Portal informático Grupo de Investigación HUM-632

http://www. proyectoprogresoarquitectura.com

Portal informático Editorial Universidad de Sevilla http://www. editorial.us.es/

(c) EDITORIAL UNIVERSIDAD DE SEVILLA, 2019.

Calle Porvenir, 27. 41013 SEVILLA. Tfs. 954487447 / 954487451

Fax 954487443. [eus4@us.es] [http://www.editorial.us.es]

(c) TEXTOS: SUS AUTORES,

(C) IMÁGENES: SUS AUTORES Y/O INSTITUCIONES

DISEÑO PORTADA:

Rosa María Añón Abajas - Amadeo Ramos Carranza

En base a la fotografía: (C) Jessica Stockholder, cortesía de la artista y de Mitchell-Innes \& Nash, Nueva York

\section{DISEÑO PLANTILLA PORTADA-CONTRAPORTADA}

Miguel Ángel de la Cova Morillo-Velarde

DISEÑO PLANTILLA MAQUETACIÓN

Maripi Rodríguez

MAQUETACIÓN

Referencias Cruzadas

CORRECCION ORTOTIPOGRÁFICA

José Antonio Duarte

ISSN (ed. impresa): 2171-6897

ISSN-e (ed. electrónica): 2173-1616

DOI: http://dx.doi.org/10.12795/ppa

DEPÓSITO LEGAL: SE-2773-2010

PERIOCIDAD DE LA REVISTA: MAYO Y NOVIEMBRE

IMPRIME: PODIPRINT

Reservados todos los derechos. Ni la totalidad ni parte de esta revista puede reproducirse o transmitirse por ningún procedimiento electrónico o mecánico, incluyendo fotocopia, grabación magnética o cualquier almacenamiento de información y sistema de recuperación, sin permiso escrito de la Editorial Universidad de Sevilla.

Las opiniones y los criterios vertidos por los autores en los artículos firmados son responsabilidad exclusiva de los mismos. 


\section{DIRECCIÓN}

Dr. Amadeo Ramos Carranza. Escuela Técnica Superior de Arquitectura. Universidad de Sevilla. España

\section{SECRETARÍA}

Dra. Rosa María Añón Abajas. Escuela Técnica Superior de Arquitectura. Universidad de Sevilla. España

\section{EQUIPO EDITORIAL \\ Edición:}

Dr. Amadeo Ramos Carranza. Escuela Técnica Superior de Arquitectura. Universidad de Sevilla. España.

Dra. Rosa María Añón Abajas. Escuela Técnica Superior de Arquitectura. Universidad de Sevilla. España.

Dr. Francisco Javier Montero Fernández. Escuela Técnica Superior de Arquitectura. Universidad de Sevilla. España.

Dra. Esther Mayoral Campa. Escuela Técnica Superior de Arquitectura. Universidad de Sevilla. España.

Dr. Miguel Ángel de la Cova Morillo-Velarde. Escuela Técnica Superior de Arquitectura. Universidad de Sevilla. España.

Dr. Germán López Mena. Escuela Técnica Superior de Arquitectura. Universidad de Sevilla. España.

Dra. Gloria Rivero Lamela. Escuela Técnica Superior de Arquitectura. Universidad de Sevilla. España.

Juan José López de la Cruz. Escuela Técnica Superior de Arquitectura. Universidad de Sevilla. España.

Guillermo Pavón Torrejón. Escuela Técnica Superior de Arquitectura. Universidad de Sevilla. España.

Externos edición (asesores):

Dr. José Altés Bustelo. Escuela Técnica Superior de Arquitectura. Universidad de Valladolid. España.

Dr. Carlos Arturo Bell Lemus. Facultad de Arquitectura. Universidad del Atlántico. Colombia.

Dr. José de Coca Leicher. Escuela Técnica Superior de Arquitectura. Universidad Politécnica de Madrid. España. Dra. Patricia de Diego Ruiz. Escuela Técnica Supeiror de Arquitectura y Geodesia. Universidad Alcalá de Heranes. España.

Dr. Alfonso del Pozo y Barajas. Escuela Técnica Superior de Arquitectura. Universidad de Sevilla. España.

Dr. Jaume J. Ferrer Fores. Escola Tècnica Superior

d'Arquitectura de Barcelona. Universitat Politècnica de Catalunya. España.

Dra. Laura MArtínez Guereñu. El School of Architecture \& Design, IE University, Madrid; Segovia. España.

Dra. Clara Mejía Vallejo. Escuela Técnica Superior de Arquitectura. Universidad Politécnica de Valencia. España.

Dra. Luz Paz Agras. Escuela Técnica Superior de Arquitectura. Universidade da Coruña. España.

Dra. Marta Sequeira. CIAUD, Faculdade de Arquitectura da Universidade de Lisboa, Portugal.

\section{SECRETARÍA TÉCNICA}

Dra. Gloria Rivero Lamela. Escuela Técnica Superior de Arquitectura. Universidad de Sevilla. España.

\section{EDITORA Y COORDINACION CONTENIDOS CIENTÍFICOS DEL NÚMERO}

Dra. Rosa María Añón Abajas. Escuela Técnica Superior de Arquitectura. Universidad de Sevilla. España.

\section{COMITÉ CIÉNTIFICO}

Dr. Carlo Azteni. DICAAR. Dipartimento di Ingegneria Civile, Ambientale e Architettura. University Of Cagliari. Italia.

Dra. Maristella Casciato. GETTY Research Institute, GETTY, Los Angeles. Estados Unidos.

Dra. Anne Marie Châtelet. École Nationale Supérieure D'Architecture de Strasbourg (ENSAS). Francia.

Dr. Jean Louis Cohen. Institute of Fine Arts, New York University. Estados Unidos.

Dra. Josefina González Cubero. Escuela Técnica Superior de Arquitectura. Universidad de Valladolid. España.

Dr. José Manuel López Peláez. Escuela Técnica Superior de Arquitectura. Universidad Politécnica de Madrid. España.

Dra. Maite Méndez Baiges. Departamento de Historia del Arte. Universidad de Málaga. España.

Dr. Dietrich C. Neumann. Brown University In Providence, Ri (John Nicholas Brown Center For Public Humanities And Cultural Heritage). Estados Unidos.

Dr. Víctor Pérez Escolano. Catedrático Historia, Teoría y Composición Arquitectónicas. Escuela Técnica Superior de Arquitectura. Universidad de Sevilla. España.

Dr. Jorge Torres Cueco. Catedrático Proyectos Arquitectónicos. Escuela Técnica Superior de Arquitectura. Universitat Politècnica de València. España.

Dr. ir. Frank van der Hoeven, TU DELFT. Architecture and the Built Environment, Netherlands

\section{CORRESPONSALES}

Pablo de Sola Montiel. The Berlage Centre for Advanced Studies in Architecture and Urban Design. Paises Bajos.

Dr. Plácido González Martínez. Tongji University Caup (College Of architectura \& Urban Planing). Shangai, China.

Patrícia Marins Farias. Faculdade de Arquitetura. Universidade Federal da Bahia. Brasil.

Dr. Daniel Movilla Vega. Umeå School of Architecture. Umeå University. Suecia.

Dr. Pablo Sendra Fernández. The Bartlett School of Planning. University College London. Inglaterra.

Alba Zarza Arribas. Centro de Estudos Arnaldo Araújo, Porto. Portugal.

Dra. María Elena Torres Pérez. Facultad de Arquitectura. Universidad Autónoma de Yucatán, Mérida. México.

\section{TEXTOS VIVOS}

Juan José López de la Cruz. Escuela Técnica Superior de Arquitectura. Universidad de Sevilla. España.

Dr. Francisco Javier Montero Fernández. Escuela Técnica Superior de Arquitectura. Universidad de Sevilla. España. Dra. Esther Mayoral Campa. Escuela Técnica Superior de Arquitectura. Universidad de Sevilla. España. 


\section{SERVICIOS DE INFORMACIÓN}

\section{CALIDAD EDITORIAL}

La Editorial Universidad de Sevilla cumple los criterios establecidos por la Comisión Nacional Evaluadora de la Actividad Investigadora para que lo publicado por el mismo sea reconocido como "de impacto" (Ministerio de Ciencia e Innovación, Resolución 18939 de 11 de noviembre de 2008 de la Presidencia de la CNEAI, Apéndice I, BOE n² 282, de 22.11.08).

La Editorial Universidad de Sevilla forma parte de la U.N.E. (Unión de Editoriales Universitarias Españolas) ajustándose al sistema de control de calidad que garantiza el prestigio e internacionalidad de sus publicaciones.

PUBLICATION QUALITY

The Editorial Universidad de Sevilla fulfils the criteria established by the National Commission for the Evaluation of Research Activity (CNEAI) so that its publications are recognised as "of impact" (Ministry of Science and Innovation, Resolution 18939 of 11 November 2008 on the Presidency of the CNEAl, Appendix I, BOE No 282, of 22.11.08).

The Editorial Universidad de Sevilla operates a quality control system which ensures the prestige and international nature of its publications, and is a member of the U.N.E. (Unión de Editoriales Universitarias Españolas-Union of Spanish University Publishers).

Los contenidos de la revista PROYECTO, PROGRESO, ARQUITECTURA aparecen en:

bases de datos: indexación
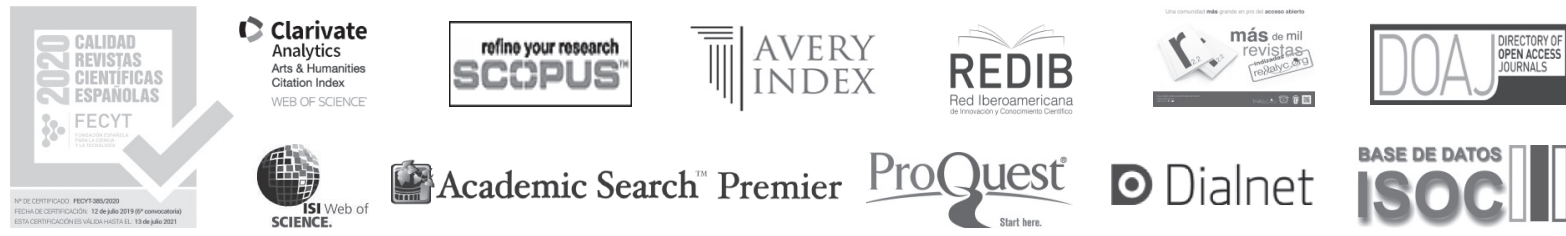

D Dialnet

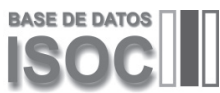

SELLO DE CALIDAD EDITORIAL FECYT 2019. RENOVADO 2020. (Cuartil C3)

WoS. Arts \& Humanities Citation Index

WoS. ESCl - Emerging Sources Citation Index

SCOPUS

AVERY. Avery Index to Architectural Periodicals

REBID. Red Iberoamericana de Innovación y Conocimiento Científico

REDALYC. Red de Revistas Científicas de América Latina y el Caribe, España y Portugal.

EBSCO. Fuente Académica Premier

EBSCO. Art Source

DOAJ, Directory of Open Access Journals

PROQUEST (Arts \& Humanities, full text)

DIALNET

ISOC (Producida por el CCHS del CSIC)

DRIJ. Directory of Research Journals Indexing

SJR (2019): 0.100, H index: 2

\section{catalogaciones: criterios de calidad}

RESH (Revistas Españolas de Ciencias Sociales y Humanidades).

Catálogos CNEAI (16 criterios de 19). ANECA (18 criterios de 21). LATINDEX (35 criterios sobre 36).

DICE (CCHS del CSIC, ANECA).

MIAR, Matriu d'Informació per a l'Avaluació de Revistes. IDCS 2018: 10,500. Campo ARQUITECTURA

CLASIFICACIÓN INTEGRADA DE REVISTAS CIENTÍFICAS (CIRC-CSIC): A

ERIHPLUS

SCIRUS, for Scientific Information.

ULRICH'S WEB, Global Serials Directory.

ACTUALIDAD IBEROAMERICANA.

\section{catálogos on-line bibliotecas notables de arquitectura:}

CLIO. Catálogo on-line. Columbia University. New York

HOLLIS. Catálogo on-line. Harvard University. Cambridge. MA

SBD. Sistema Bibliotecario e Documentale. Instituto Universitario di Architettura di Venezia

OPAC. Servizi Bibliotecari di Ateneo. Biblioteca Centrale. Politecnico di Milano

COPAC. Catálogo colectivo (Reino Unido)

SUDOC. Catálogo colectivo (Francia)

ZBD. Catálogo colectivo (Alemania)

REBIUN. Catálogo colectivo (España)

OCLC. WorldCat (Mundial) 
EVALUACIÓN EXTERNA POR PARES Y ANÓNIMA.

El Consejo Editorial remitirá el artículo a dos expertos revisores anónimos dentro del campo específico de investigación y crítica de arquitectura, según el modelo doble ciego.

El director de la revista comunicará a los autores el resultado motivado de la evaluación por correo electrónico, en la dirección que éstos hayan utilizado para enviar el artículo. El director comunicará al autor principal el resultado de la revisión (publicación sin cambios; publicación con correcciones menores; publicación con correcciones importantes; no aconsejable para su publicación), así como las observaciones y comentarios de los revisores.

Si el manuscrito ha sido aceptado con modificaciones, los autores deberán reenviar una nueva versión del artículo, atendiendo a las demandas y sugerencias de los evaluadores externos. Los artículos con correcciones importantes serán remitidos al Consejo Asesor para verificar la validez de las modificaciones efectuadas por el autor. Los autores pueden aportar también una carta al Consejo Editorial en la que indicarán el contenido de las modificaciones del artículo. Los artículos con correcciones importantes serán remitidos al Consejo Asesor para verificar la validez de las modificaciones efectuadas por el autor.

\section{DECLARACIÓN ÉTICA SOBRE PUBLICACIÓN Y MALAS PRÁCTICAS}

La revista PROYECTO, PROGRESO, ARQUITECTURA (PPA) está comprometida con la comunidad académica en garantizar la ética y calidad de los artículos publicados. Nuestra revista tiene como referencia el Código de Conducta y Buenas Prácticas que, para editores de revistas científicas, define el COMITÉ DE ÉTICA DE PUBLICACIONES (COPE).

Así nuestra revista garantiza la adecuada respuesta a las necesidades de los lectores y autores, asegurando la calidad de lo publicado, protegiendo y respetando el contenido de los artículos y la integridad de los mismo. El Consejo Editorial se compromete a publicar las correcciones, aclaraciones, retracciones y disculpas cuando sea preciso.

En cumplimiento de estas buenas prácticas, la revista PPA tiene publicado el sistema de arbitraje que sigue para la selección de artículos así como los criterios de evaluación que deben aplicar los evaluadores externos -anónimos y por pares, ajenos al Consejo Editorial-. La revista PPA mantiene actualizados estos criterios, basados exclusivamente en la relevancia científica del artículo, originalidad, claridad y pertinencia del trabajo presentado.

Nuestra revista garantiza en todo momento la condifencialidad del proceso de evaluación: el anonimato de los evaluadores y de los autores; el contenido evaluado; los informes razonados emitidos por los evaluadores y cualquier otra comunicación emitida por los consejos Editorial, Asesor y Científico si así procediese.

Igualmente quedan afectados de la máxima confidencialidad las posibles aclaraciones, reclamaciones o quejas que un autor desee remitir a los comités de la revista o a los evaluadores del artículo.

La revista PROYECTO, PROGRESO, ARQUITECTURA (PPA) declara su compromiso por el respeto e integridad de los trabajos ya publicados. Por esta razón, el plagio está estrictamente prohibido y los textos que se identifiquen como plagio o su contenido sea fraudulento, serán eliminados o no publicados por la revista PPA. La revista actuará en estos casos con la mayor celeridad posible. Al aceptar los términos y acuerdos expresados por nuestra revista, los autores han de garantizar que el artículo y los materiales asociados a él son originales o no infringen derechos de autor. También los autores tienen que justificar que, en caso de una autoría compartida, hubo un consenso pleno de todos los autores afectados y que no ha sido presentado ni publicado con anterioridad en otro medio de difusión.

\section{EXTERNAL ANONYMOUS PEER REVIEW.}

Editorial Board will be sent to two anonymous experts, within the specific field of architectural investigation and critique, for a double blind review.

The Director of the journal will communicate the result of the reviewers evaluations to the authors by electronic mail, to the address used to send the article. The Director will communicate the result of the review (publication without changes; publication with minor corrections; publication with significant corrections; its publication is not advisable), as well as the observations and comments of the reviewers, to the main author.

If the manuscript has been accepted with modifications, the authors will have to resubmit a new version of the article, addressing the requirements and suggestions of the external reviewers. The articles with corrections will be sent to Advisory Board for verification of the validity of the modifications made by the author. The authors can also send a letter to the Editorial Board, in which they will indicate the content of the modifications of the article.

\section{ETHICS STATEMENT ON PUBLICATION AND BAD PRACTICES} PROYECTO, PROGRESO ARQUITECTURA (PPA) makes a commitment to the academic community by ensuring the ethics and quality of its published articles. As a benchmark, our journal uses the Code of Conduct and Good Practices which, for scientific journals, is defined for editors by the PUBLICATION ETHICS COMMITTEE (COPE).

Our journal thereby guarantees an appropriate response to the needs of readers and authors, ensuring the quality of the published work, protecting and respecting the content and integrity of the articles. The Editorial Board will publish corrections, clarifications, retractions and apologies when necessary.

In compliance with these best practices, PPA has published the arbitration system that is followed for the selection of articles as well as the evaluation criteria to be applied by the anonymous, external peer-reviewers. PPA keeps these criteria current, based solely on the scientific importance, the originality, clarity and relevance of the presented article.

Our journal guarantees the confidentiality of the evaluation process at all times: the anonymity of the reviewers and authors; the reviewed content; the reasoned report issued by the reviewers and any other communication issued by the editorial, advisory and scientific boards as required.

Equally, the strictest confidentiality applies to possible clarifications, claims or complaints that an author may wish to refer to the journal's committees or the article reviewers.

PROYECTO, PROGRESO ARQUITECTURA (PPA) declares its commitment to the respect and integrity of work already published. For this reason, plagiarism is strictly prohibited and texts that are identified as being plagiarized, or having fraudulent content, will be eliminated or not published in PPA. The journal will act as quickly as possible in such cases. In accepting the terms and conditions expressed by our journal, authors must guarantee that the article and the materials associated with it are original and do not infringe copyright. The authors will also have to warrant that, in the case of joint authorship, there has been full consensus of all authors concerned and that the article has not been submitted to, or previously published in, any other media. 
EVALUADORES EXTERNOS (publicación cada cuatro números, dos años). NÚMEROS 21 a 24 (incluidos)

Álvarez Álvarez, Darío. Catedrático de Universidad / Departamento de Teoría de la Arquitectura y Proyectos Arquitectónicos / ETS Arquitectura / Universidad de Valladolid / España.

Arrieta Berdasco, Valentín. Doctor Arquitecto, Profesor Asociado / Departamento de Teoría de la Arquitectura y Proyectos Arquitectónicos / ETS Arquitectura / Universidad de Valladolid / España.

Bardí i Milá, Berta. Profesora Asociada doctora / / Departamento de Projectes Arquitectònics / ETS Arquitectura / Universitat Politècnica de Catalunya, Barcelona Tech / España.

Bergera Serrano, Iñaki. Titular de Universidad / Unidad Predepartamental de Arquitectura / Área Proyectos Arquitectónicos / El y Arquitectura / Universidad de Zaragoza / España.

Bobbink, Inge. Dr. ir. Architecture / Section of landscape architecture / TU Delft / Países Bajos.

Burriel Bielza, Luis. Profesor Titular / École Nationale Supérieure d'Architecture de Paris-Belleville / Francia

Calatrava Escobar, Juan. Catedrático de Universidad / Departamento de Construcciones Arquitectónicas / ETS Arquitectura / Universidad de Granada / España.

Castellanos Gómez, Raúl. Titular de Universidad / Departamento de Proyectos Arquitectónicos / ETS Arquitectura / Universidad Politécnica de Valencia / España.

Centellas Soler, Miguel. Titular de Universidad / Departamento de Arquitectura y Tecnología de la Edificación / ETS Arquitectura y Edificación / Universidad Politécnica de Cartagena / España.

Chías Navarro, Pilar. Catedrática de Universidad / Departamento de Arquitectura / ETS Arquitectura y Geodesia / Universidad de Alcalá de Henares / España.

de Diego Ruiz, Patricia. Doctora arquitecta, Profesora Asociada / Departamento de Arquitectura / ETS Arquitectura y Geodesia / Universidad de Alcalá de Henares / España.

de la Iglesia Salgado, Félix. Profesor Contratado Doctor / Departamento de Proyectos Arquitectónicos / ETS Arquitectura / Universidad de Sevilla / España.

de la O Cabrera, Manuel Rodrigo. Doctor Arquitecto, Profesor Asociado / Departamento de Composición Arquitectónica / ETS Arquitectura / Universidad Politécnica de Madrid / España.

Delgado Orusco, Eduardo. Profesor Ayudante Doctor / Departamento de Arquitectura. Área de Proyectos Arquitectónicos / Escuela de Ingeniería y Arquitectura / Universidad de Zaragoza / España.

Deltell Pastor, Juan. Titular de Universidad / Departamento de Proyectos Arquitectónicos / ETS Arquitectura / Universitat Politècnica de València / España.

Diañez Rubio, Pablo. Titular de Universidad / Departamento de Proyectos Arquitectónicos / ETS Arquitectura / Universidad de Sevilla / España.

Díaz Segura, Alfonso. Titular de Universidad / Proyectos, Teoría y Técnica del Diseño y la Arquitectura / ETS Arquitectura / Universidad CEU Cardenal Herrera, Valencia / España.

Domingo Calabuig, Débora. Titular de Universidad / Departamento de Proyectos Arquitectónicos / ETS Arquitectura / Universidad Politécnica de Valencia / España.

Fernández Fariña, Almudena. Profesora Contratada Doctora / Departamento de Pintura / Facultad de Bellas Artes / Universidad de Vigo / España.

Fernández-Trapa de Isasi, Justo. Catedrático de Universidad / Departamento de Proyectos Arquitectónicos / ETS Arquitectura / Universidad Politécnica de Madrid / España.

García Escudero, Daniel. Dr. Arquitecto, Profesor Lector / Departamento de Projectes Arquitectònics / ETS Arquitectura / Universitat Politècnica de Catalunya, Barcelona Tech / España.

González Cubero, Josefina. Titular de Universidad / Departamento de Teoría de la Arquitectura y Proyectos Arquitectónicos / ETS Arquitectura / Universidad de Valladolid / España.

González Fraile, Eduardo. Catedrático de Universidad / Departamento de Teoría de la Arquitectura y Proyectos Arquitectónicos / ETS Arquitectura / Universidad de Valladolid / España.

Gorostiza López, Jorge. Doctor Arquitecto. Cineasta / España.

Hernández Moreno, Silverio. Profesor investigador titular / Facultad de Arquitectura y Diseño / Universidad Autónoma del Estado de México / México.

Labarta Aizpún, Carlos. Titular de Universidad / Unidad Predepartamental de Arquitectura / Área Proyectos Arquitectónicos / Escuela de Ingeniería y Arquitectura / Universidad de Zaragoza / España.

Lizondo Sevilla, Laura. Titular de Universidad / Departamento de Proyectos Arquitectónicos / ETS Arquitectura / Universidad Politécnica de Valencia / España.

Llopis Verdú, Jorge. Catedrático de Universidad / Departamento de Expresión Gráfica Arquitectónica / ETS Arquitectura / Universidad Politécnica de Valencia / España.

López Bahut, Emma. Profesora Contratada Doctora / Departamento de Proyectos arquitectónicos, Urbanismo y Composición / ETS Arquitectura / Universidade da Coruña / España.

López Fernández, Andrés. Titular de Universidad / Departamento de Proyectos Arquitectónicos / ETS Arquitectura / Universidad de Sevilla / España.

Loren Méndez, Mar. Catedrática de Universidad / Departamento de Historia, Teoría y Composición Arquitectónica / ETS Arquitectura / Universidad de Sevilla / España.

Maino Ansaldo, Sandro. Doctor Arquitecto / Departamento Arquitectura / Universidad Técnica Federico Santa María / Chile.

Mària i Serrano, Magda. Profesora Contratada Doctor / Departamento de Projectes Arquitectònics / ETS Arquitectura del Vallès / Universitat Politècnica de Catalunya / España

Marson, Anna. Profesora Ordinario / Dipartimento di culture del progetto / Istituto Universitario di Architettura di Venezia / Università di Venezia / Italia.

Martínez Díaz, Ángel. Titular de Universidad / Departamento de Ideación Gráfica Arquitectónica / ETS Arquitectura / Universidad Politécnica de Madrid / España. 
Mejía Vallejo, Clara. Titular de Universidad / Departamento de Proyectos Arquitectónicos / ETS Arquitectura / Universidad Politécnica de Valencia / España.

Mercader Moyano, Pilar. Titular de Universidad / Departamento de Proyectos Arquitectónicos / ETS Arquitectura / Universidad de Sevilla / España.

Mercé Hospital, José María. Catedrático de Universidad / Departamento de Proyectos Arquitectónicos / ETS Arquitectura y Geodesia / Universidad Alcalá de Henares / España.

Merí de la Maza, Ricardo. Titular de Universidad / Departamento de Proyectos Arquitectónicos / ETS Arquitectura / Universidad Politécnica de Valencia / España.

Mestre Martínez, Nieves. Doctora arquitecta, Profesora Asociada / Departamento de Proyectos Arquitectónicos / ETS Arquitectura / Universidad Politécnica de Madrid / España.

Millán Gómez, Antonio. Catedrático de Universidad / Departamento d’Expressió Gràfica Arquitectònica I / ETS Arquitectura del Vallès / Universitat Politècnica de Catalunya / España.

Moreno Pérez, José Ramón. Titular de Universidad / Dpto. Historia, Teoría y Composición Arquitectónica / ETS Arquitectura / Universidad de Sevilla / España.

Nijhuis, Steffen. Associate Professor. Head of Landscape Architecture Research / Section of Landscape Architecture / Department of Urbanism / Faculty of Architecture and the Built Environment / TU Delft / Países Bajos.

Ojeda Rivera, Juan Francisco. Catedrático de Universidad / Departamento de Geografía, Historia y Filosofía / Universidad Pablo de Olavide, Sevilla / España.

Paz-Agras, Luz. Profesora Ayudante doctor / Departamento de Proyectos Arquitectónicos, Urbanismo y Composición. Área de Composición Arquitectónica / ETS Arquitectura / Universidad de A Coruña / España

Pérez Moreno, Lucía C. Titular de Universidad / Departamento de Arquitectura. Área de Composición Arquitectónica / Escuela de Ingeniería y Arquitectura / Universidad de Zaragoza / España.

Pina Lupiañez, Rafael. Profesor Contratado Doctor / Departamento de Proyectos Arquitectónicos / ETS Arquitectura / Universidad Politécnica de Madrid / España

Oliverira do Nascimento, Francisco. Professor Auxiliar / Faculdade de Arquitetura / Universidade de Lisboa / Portugal.

Rovira Llobera, Teresa. Titular de Universidad / Departamento de Projectes Arquitectònics / ETS Arquitectura / Universitat Politècnica de Catalunya, Barcelona Tech / España.

Ruiz Rosa, José Antonio. Catedrático de Universidad / Departamento de Expresión Gráfica Arquitectónica / ETS Arquitectura / Universidad de Sevilla. / España.

Sabaté Bel, Joaquín. Catedrático de Universidad / Departamento de Urbanismo y Ordenación del Territorio / ETS Arquitectura / Universitat Politècnica de Catalunya, Barcelona Tech / España

Sainz Gutiérrez Victoriano. Titular de Universidad / Departamento de Urbanismo y Ordenación del Territorio / ETS Arquitectura / Universidad de Sevilla / España

Sánchez Lampreave, Ricardo. Profesor Titular / Área de Composición Arquitectónica / Escuela de Ingeniería y Arquitectura / Universidad de Zaragoza / España.

Sambricio R. Echegaray, Carlos. Catedrático de Universidad / Departamento de Composición Arquitectónica / ETS Arquitectura / Universidad Politécnica de Madrid / España. / España.

Santamarina-Macho, Carlos. España. Doctor Arquitecto, Profesor Asociado / Departamento de Teoría de la Arquitectura y Proyectos Arquitectónicos / ETS Arquitectura / Universidad de Valladolid / España

Senra Fernández-Miranda, Ignacio. Doctor Arquitecto, Profesor Asociado / Departamento de Proyectos Arquitectónicos / ETS Arquitectura / Universidad Politécnica de Madrid / España.

Sentieri Omarrementeria, Carla. Titular de Universidad / Departamento de Proyectos Arquitectónicos / ETS Arquitectura / Universidad Politécnica de Valencia / España.

Sequeira Marta. Professora doctora / ISCTE - Instituto Universitário de Lisboa/ Universidade Autónoma de Lisboa / CIAUD - Faculdade de Arquitectura da Universidade de Lisboa / Portugal.

Sola Alonso, José Ramón. España. Profesor Contratado Doctor / Departamento de Teoría de la Arquitectura y Proyectos Arquitectónicos / ETS Arquitectura / Universidad de Valladolid / España.

Trillo Martínez, Valentín. Doctor arquitecto Profesor Asociado / Departamento de Proyectos Arquitectónicos / ETS Arquitectura / Universidad de Sevilla / España.

Villalobos Alonso, Daniel. Titular de Universidad / Departamento de Teoría de la Arquitectura y Proyectos Arquitectónicos / ETS Arquitectura / Universidad de Valladolid / España.

Verde Zein, Ruth. Doctora arquitecto e investigadora / Facultad de Arquitectura y Urbanismo / Universidad Presbiteriana Mackenzie. São Paulo / Brasil.

\section{ESTADÍSTICAS PUBLICACIÓN (publicación cada cuatro números, dos años). NÚMEROS 1 a 20 (incluidos)}

Total artículos recibidos: 546

Total artículos publicados: 199 (36,45\%)

Total artículos rechazados: $347(63,55 \%)$

Total artículos publicados de autores pertenecientes a los diferentes consejos o comités organizadores de la revista y Grupo de Investigación "proyecto, progreso, arquitectura"(endogamia): 20 (10,05\%)

Total artículos publicados de autores pertenecientes a la Universidad de Sevilla: 53 (26,63\%)

Total artículos publicados de autores externos a los diferentes consejos o comités organizadores de la revista y Grupo de Investigación

"proyecto, progreso, arquitectura": 179 (89,95\%)

Total artículos publicados de autores extranjeros: 17 (8,54\%) 


\section{arquitecturas ampliadas}

índice

editorial

DESPEJAR LA ARQUITECTURA, LIBERAR EL ESPACIO Y AMPLIAR CONCEPTOS / UNCLUTTER ARCHITECTURE, FREE UP SPACE AND EXPAND CONCEPTS

Rosa María Añón-Abajas - (DOI: http://dx.doi.org/10.12795/ppa.2021.i24.10)

entre líneas

PAISAJES ARQUITECTÓNICOS Y MEMORIAS DE LA CIUDAD / ARCHITECTURAL LANDSCAPES AND MEMORIES OF THE CITY

Darío Álvarez Álvarez - (D0l: http://dx.doi.org/10.12795/ppa.2021.i24.01)

artículos

LINAZASORO EN REIMS. EL ESPACIO PÚBLICO COMO MEMORIA DEL LUGAR / LINAZASORO IN REIMS. THE PUBLIC SPACE AS THE MEMORY OF THE PLACE

Victoriano Sainz Gutiérrez - (DOl: http://dx.doi.org/10.12795/ppa.2021.i24.02)

EDITAR VS. CONSTRUIR: UNA ECOLOGÍA DE LO INVISIBLE. AMPLIFICAR LA COMPRENSIÓN DE LAS TÉCNICAS DE PROYECTO / EDITING VS. BUILDING: AN ECOLOGY OF THE INVISIBLE. AMPLIFYING THE UNDERSTANDING OF ARCHITECTURAL DESIGN TECHNIQUES

Paula Victoria Álvarez Benítez - (DOI: http://dx.doi.org/10.12795/ppa.2021.i24.03)

CUANDO LA PINTURA AMPLÍA LA ARQUITECTURA: INTERVENCIONES REALIZADAS EN EL ESPACIO PÚBLICO / WHEN PAINTING ENHANCES ARCHITECTURE: INTERVENTIONS IN A PUBLIC SETTING Aurora Alcaide-Ramírez; Ana Ruiz-Abellón - (D0l: http://dx.doi.org/10.12795/ppa.2021.i24.04)

UN EDIFICIO INVISIBLE. NUEVO AULARIO DE LA FACULTAD DE DERECHO DE LA UNIVERSIDAD DE ZARAGOZA (1983-1996) / AN INVISIBLE BUILDING. NEW LECTURE ROOM BUILDING OF THE FACULTY OF LAW OF THE UNIVERSITY OF ZARAGOZA (1983-1996)

Luis Miguel Lus-Arana; Lucía Carmen Pérez-Moreno - (D0l: http://dx.doi.org/10.12795/ppa.2021.i24.05)

ARQUITECTURAS AMPLIADAS. EL PABELLÓN DE EXPOSICIONES EN LA CASA DE CAMPO DE MADRID) / EXPANDED ARCHITECTURES. THE EXHIBITION PAVILION AT THE CASA DE CAMPO IN MADRID José de Coca Leicher - (DOI: http://dx.doi.org/10.12795/ppa.2021.i24.06)

reseña bibliográfica TEXTOS VIVOS

RAFAEL MONEO VALLÉS: LA VIDA DE LOS EDIFICIOS. LA MEZQUITA DE CÓRDOBA, LA LONJA DE SEVILLA Y UN CARMEN EN GRANADA

Víctor Pérez Escolano - (DOl: http://dx.doi.org/10.12795/ppa.2021.i24.07)

FRANCISCO DE GRACIA: CONSTRUIR EN LO CONSTRUIDO. LA ARQUITECTURA COMO MODIFICACIÓN

Pablo Diañez Rubio - (DOl: http://dx.doi.org/10.12795/ppa.2020.i24.08)

FRÉDÉRIC DRUOT, ANNE LACATON \& JEAN-PHILIPPE VASSAL PLUS: LA VIVIENDA COLECTIVA. TERRITORIO DE EXCEPCIÓN

Javier Terrados Cepeda - (DOI: http://dx.doi.org/10.12795/ppa.2021.i24.09) 


\title{
UN EDIFICIO INVISIBLE. NUEVO AULARIO DE LA FACULTAD DE DERECHO DE LA UNIVERSIDAD DE ZARAGOZA (1983-1996)
}

\author{
AN INVISIBLE BUILDING. NEW LECTURE ROOM BUILDING OF THE FACULTY OF LAW OF \\ THE UNIVERSITY OF ZARAGOZA (1983-1996)
}

Luis Miguel Lus-Arana (https://orcid.org/0000-0001-5826-2642)

Lucía C. Pérez-Moreno (https://orcid.org/0000-0002-6303-1950)

RESUMEN En 1983, el equipo formado por Saturnino Cisneros Lacruz, Juan Antonio Carmona Mateu, Manuel Fernández Ramírez e Isabel García Elorza comenzaba la construcción del proyecto de ampliación de la Facultad de Derecho de la Universidad de Zaragoza. La necesidad de construir un nuevo aulario surgía de la mano del progresivo aumento de estudiantes de Derecho que se estaban dando en España desde los años sesenta. La autonomía formal, junto a la contundente geometría circular de este nuevo aulario, es muestra de la intención de sus autores por romper, de manera respetuosa, con la arquitectura racionalista regionalista, que Regino Borobio y José Beltrán Navarro habían construido en los primeros años cuarenta y que había dominado la ordenación de la Ciudad Universitaria de la ciudad de Zaragoza. Con concomitancias formales aaltianas y ecos nacionales a las arquitecturas de Fernando Higueras de los años sesenta, esta ampliación proponía una arquitectura tardomoderna afín al brutalismo de Alison y Peter Smithson donde los espacios de relación e intercambio social se tornaron aspectos esenciales y definitorios del proyecto. Esta ampliación conjuga autonomía con subordinación, tanto física como funcional, con respeto al edificio original, generando una pieza que combina rotundidad volumétrica y amabilidad para con el usuario, y cuya escala percibida desdice su enorme volumen construido.

PALABRAS CLAVE tardomodernidad; brutalismo; Aragón; arquitectura española; Isabel García Elorza; arquitectura educativa

SUMMARY In 1983, the team formed by Saturnino Cisneros Lacruz, Juan Antonio Carmona Mateu, Manuel Fernández Ramírez and Isabel García Elorza began the construction of the extension to the Faculty of Law of the University of Zaragoza. The need to build a new lecture room building arose from the progressive increase in law students that had been taking place in Spain since the 1960s. The new building's formal autonomy, together with its bold circular geometry, testifies to the authors' will to break, in a respectful way, with the regionalist rationalist architecture that Regino Borobio and José Beltrán Navarro had built in the early 1940s, which had dominated the development of the University Campus of the city of Zaragoza. With formal Aaltian concomitances and echoes of Fernando Higueras's architecture during the 1960s in the national context, this extension proposed a late-modern architecture akin to Alison and Peter Smithson's Brutalism, where the spaces of relation and social exchange became both essential and defining aspects of the project. The extension combines autonomy with subordination, both physical and functional, to the original building, generating a piece that combines volumetric rotundity with friendliness towards the user, a piece whose perceived scale belittles its enormous mass.

KEYWORDS late modernity; Brutalism; Aragon; Spanish architecture; Isabel García Elorza; educational architecture 
$E$ n 1983, la Universidad de Zaragoza comenzó la construcción de la ampliación de su Facultad de Derecho ${ }^{1}$. Este proyecto sería el último de un conjunto de nuevos edificios construidos en la Ciudad Universitaria -actual Campus de San Francisco- entre 1969 y 1989. La acuciante necesidad de esta ampliación derivaba de los importantes cambios educativos que se estaban dando en España desde el tardofranquismo y que la transición hacia la democracia comenzaba a consolidar. Desde los años sesenta, el número de personas que querían formarse para ejercer una profesión jurídica mostraba un crecimiento considerable y sostenido con un incremento en el número de estudiantes en el que destacaba, además, la progresión sin precedentes de la presencia femenina en las aulas. En el caso de la Facultad de Derecho de Zaragoza, este incremento generalizado se plasmó en la necesidad de construir un nuevo aulario que pudiese albergar a unos 2800 nuevos alumnos y alumnas.
El proyecto del nuevo aulario es obra de un equipo de arquitectos formado por Saturnino Cisneros Lacruz, Juan Antonio Carmona Mateu, Manuel Fernández Ramírez e Isabel García Elorza -una de las primeras arquitectas en ejercer en la comunidad autónoma de Aragón²-. Su proyecto para este nuevo aulario muestra una respetuosa ruptura con la mezcla de modernidad y regionalismo propia de la España de la autarquía que había dominado la arquitectura de la Ciudad Universitaria durante el régimen. Alejado tanto de la hierática monumentalidad de los edificios de los años 30 y 40 como del juego escultórico de las composiciones de paralelepípedos de las obras posteriores, el edificio nace del equilibrio entre la autonomía compositiva y la búsqueda de la integración funcional entre lo nuevo y lo existente. Si en los edificios originales la presencia exterior había sido fundamental en la definición urbana del campus, en el nuevo aulario primaba la creación de un mundo interior: un conjunto de espacios arquitectónicos sin jerarquías, donde los espacios

1. Esta obra sería la primera promovida por la propia universidad en virtud de la Ley de Reforma Universitaria aprobada ese mismo año. GONZÁLEZ MARTíNEZ, Carlos; BIEL IBÁÑ̃EZ, Pilar; HERNÁNDEZ MARTíNEZ, Ascensión, coords. La Universidad de Zaragoza: arquitectura y ciudad. Zaragoza: Universidad de Zaragoza, 2008, pp. 99-161.

2. En 1983, Aragón contaba únicamente con 16 arquitectas registradas en su Colegio Oficial de Arquitectos, de las cuales Isabel García Elorza había sido la tercera en colegiarse en 1971. La primera arquitecta colegiada en Aragón data de 1968 y fue Elvira Adiego, titulada por la Escuela de Arquitectura de Madrid. 


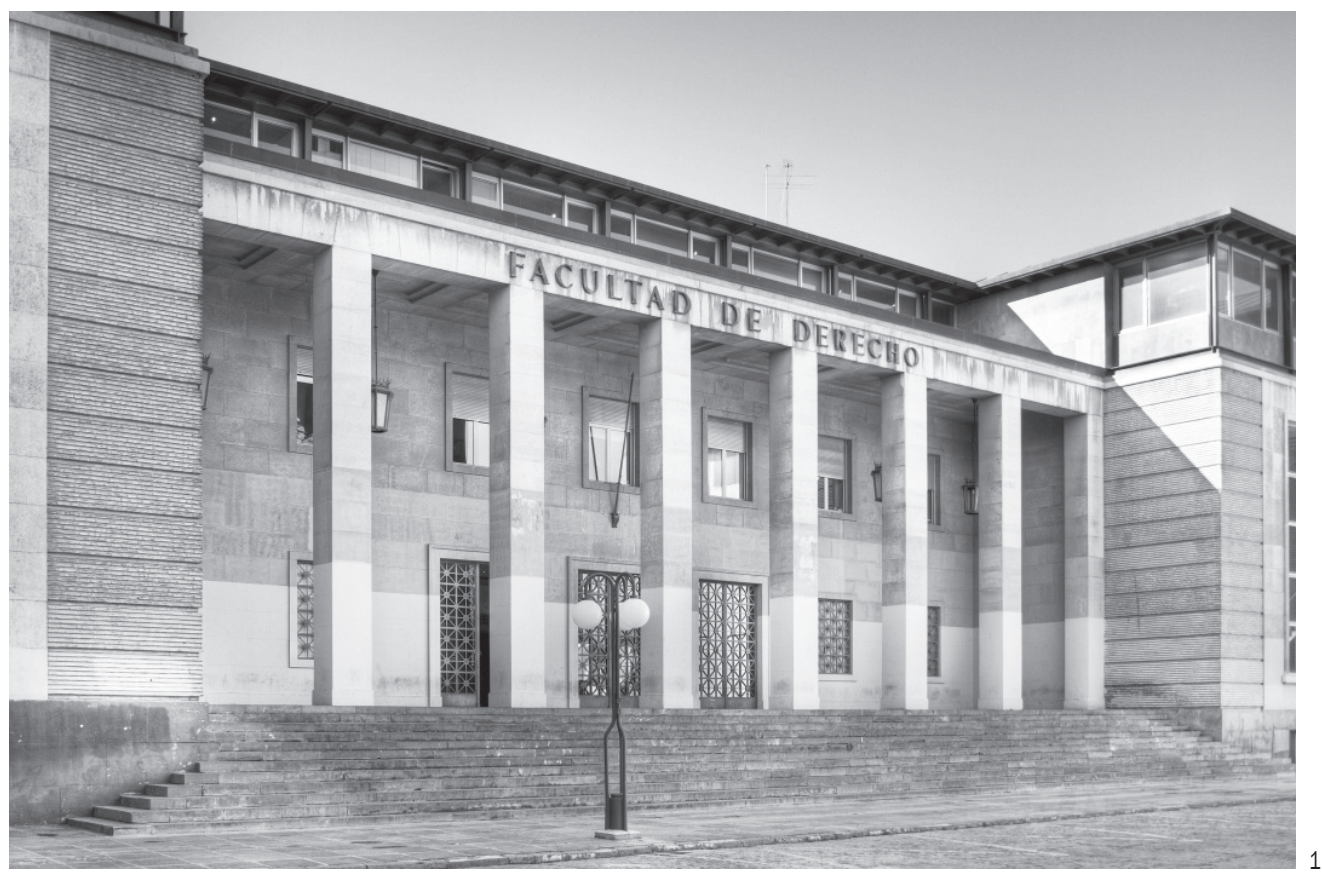

de relación vital entre alumnos y alumnas, profesorado y personal de administración pasaban a ser protagonistas.

\section{LA FACULTAD DE DERECHO Y LA CIUDAD UNIVERSITARIA DE ZARAGOZA}

El edificio original de la Facultad de Derecho (figura 1) había sido construido y reformado entre 1939 y 1945 por Regino Borobio y José Beltrán Navarro ${ }^{3}$, seis años después de que los mismos autores ganaran el concurso de anteproyectos para la ordenación de la Ciudad Universitaria de Zaragoza (figura 2). Este sería en realidad el segundo edificio realizado por ambos en el campus: en 1935 habían diseñado la Facultad de Filosofía y Letras, y entre 1954 y 1966 terminarían de acotar la plaza central de la Ciudad Universitaria con la Facultad de Ciencias. Con ella, este espacio conformaría una $\mathrm{C}$ abierta hacia el noreste y presidida por la Facultad de Derecho, que sería cerrada en 1973, con la terminación del edificio Interfacultades y el pabellón anexo de Rectorado de Isidoro Garasa (1970-73), que marcaría el inicio de la segunda gran expansión del campus desde finales de los 60 y durante toda la década de 1970.
Derecho y sus dos facultades hermanas, junto con otros edificios construidos por Borobio y Beltrán Navarro, como el Colegio Mayor Universitario Pedro Cerbuna (1942-1950) o la residencia de profesores (1964-69), situadas en la parcela adyacente al nuevo aulario, establecerían los parámetros que definirían la arquitectura de la Ciudad Universitaria. Con carácter general, las edificaciones del campus se caracterizarán por un racionalismo moderno heredero de la composición Beaux Arts, con plantas ordenadas según una retícula cartesiana y edificios formados por la concatenación de elementos rectangulares. El aspecto exterior vendría marcado por la severidad de unos alzados de huecos regulares y el uso masivo del ladrillo en las envolventes. Ambos, junto con las cubiertas inclinadas de los primeros edificios, respondían a los rasgos que caracterizarían a la arquitectura de la autarquía española en los años cuarenta ${ }^{4}$ que, en Zaragoza, recogía tanto la tradición constructiva aragonesa como la propia intrahistoria de la universidad, ligada durante el cambio de siglo a la obra del arquitecto Ricardo Magdalena y su recuperación de la arquitectura del Renacimiento aragonés ${ }^{5}$.

3. VÁZQUEZ ASTORGA, Mónica. José Borobio. Su aportación a la arquitectura moderna. Zaragoza: Delegación del Gobierno en Aragón. Caja de Ahorros de la Inmaculada, 2007, pp. 228-234.

4. BIEL IBÁÑ̃EZ, María Pilar. Autarquía y arquitectura industrial en Zaragoza. En: Artigrama, 2003, n. ํ 18, pp. 527-548. DIÉGUEZ, Sofía. Arquitectura y urbanismo durante la autarquía. En: Antonio BONET CORREA (coord.), Arte del franquismo. Madrid: Cátedra, 1981, pp. 47-76.

5. Precisamente, el actual Paraninfo de la Universidad de Zaragoza, construido por Ricardo Magdalena como Hospital Clínico o Facultad de Medicina y Ciencias entre 1886 y 1893, sería objeto de una sustancial reforma por parte de Borobio y Beltrán Navarro entre 1957 y 1963. 
1. Facultad de Derecho de la Universidad de Zaragoza, primer edificio de Regino Borobio y José Beltrán Navarro.

2. Plano general de la Ciudad Universitaria de Zaragoza. Relación de edificios y facultades del campus: 1. Facultad de Filosofía y Letras; 2. Biblioteca de Humanidades María Moliner; 3. Residencia de profesores; 4. Facultad de Derecho; 5. Instituto de Ciencias de la Educación; 6. Colegio Mayor Santa Isabel; 7. Facultad de Educación; 8. Facultad de Ciencias; 9. Facultad de Ciencias (ampliación); 10. Facultad de Ciencias: Edificio de Matemáticas; 11. Facultad de Geología; 12. Edificio Interfacultades; 13. Pabellón Polideportivo Universitario SAD; 14. Colegio Mayor Universitario Pedro Cerbuna; 15. Facultad de Medicina Edificio A; 16. CIBA. Centro de Investigación Biomédica de Aragón; 17. Facultad de Medicina; 18. Facultad de Ciencias Sociales y del Trabajo; 19. Facultad de Ciencias de la Salud.

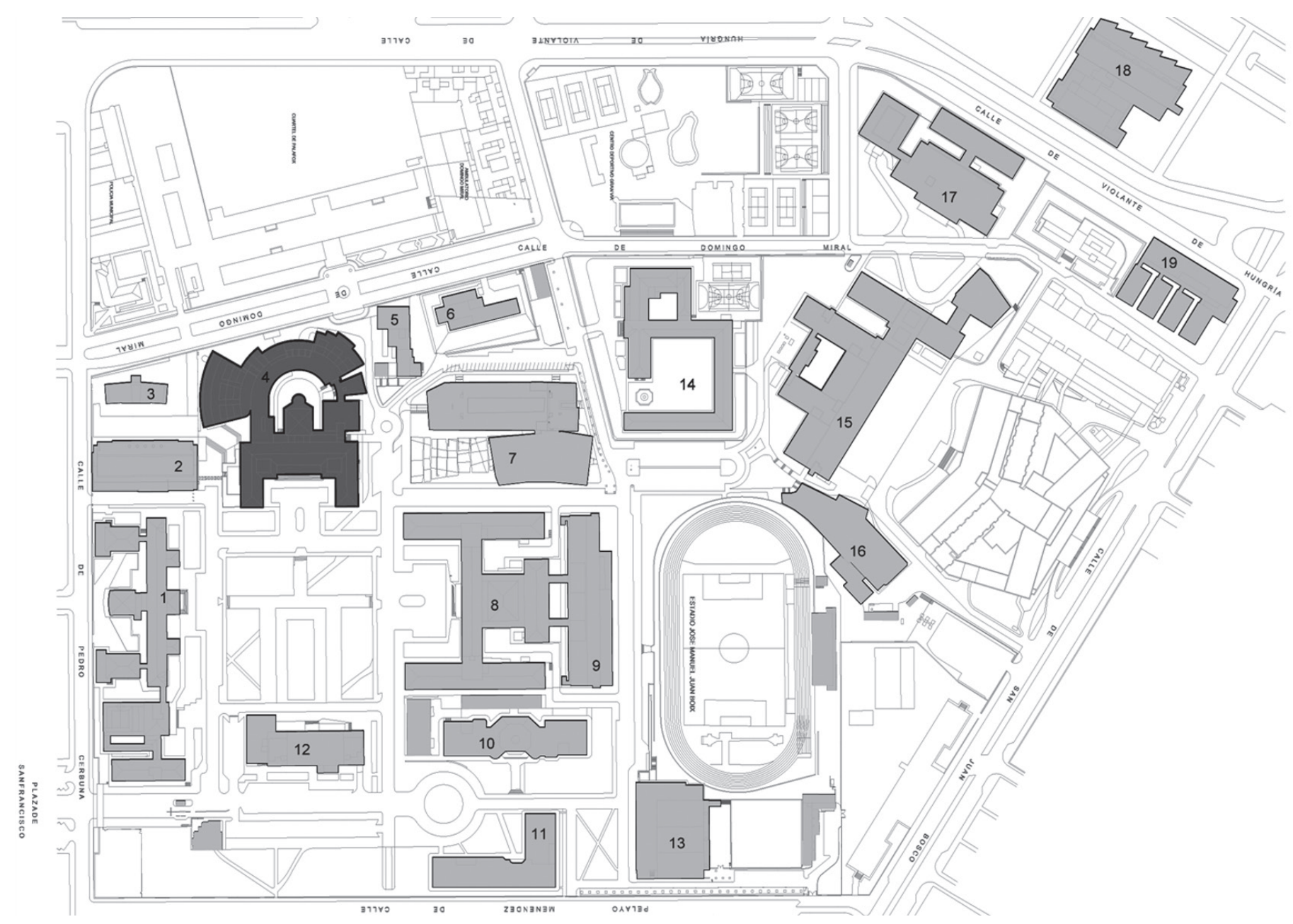

El primer edificio de la Facultad de Derecho adoptaba un esquema en $U$ abierta a la gran plaza central ajardinada a la que dan frente la Facultad de Filosofía y Letras al este, el Edificio de Ciencias al oeste y el edificio de Interfacultades al norte. La pieza se organiza simétricamente con el acceso principal situado en la mitad de su lado largo, marcando el eje de simetría, y al cual se accede a través de una gran escalinata propia de la arquitectura beauxartiana (figura 1). Los diferentes espacios interiores se organizaban de manera lineal a lo largo de la pieza en $\cup$ y son accesibles a través de un ancho y homogéneo espacio corredor que actúa al mismo tiempo de vestíbulo de entrada en la planta baja como de espacio de reunión y de distribución a aulas y espacios de administración en planta sótano, principal y primera (figura 3).

A este rígido esquema compositivo en $U$ se le añade un elemento más al sur, el aula magna; un volumen casi cúbico situado en el eje de simetría enfrentado a la gran escalinata principal y al espacio del vestíbulo central. Esta configuración se mantendría inalterada hasta octubre de 1970, momento en que Borobio y Beltrán proyectarían la primera de las ampliaciones de la facultad, un nuevo 

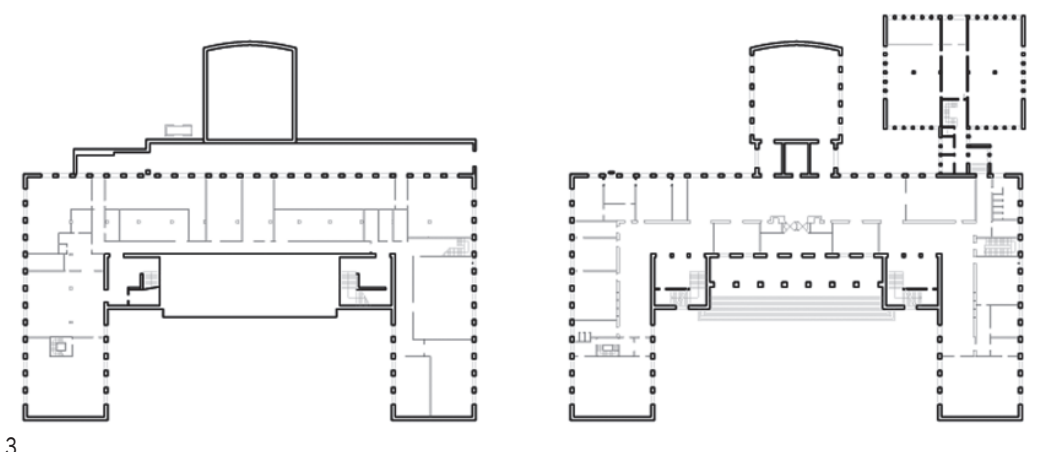

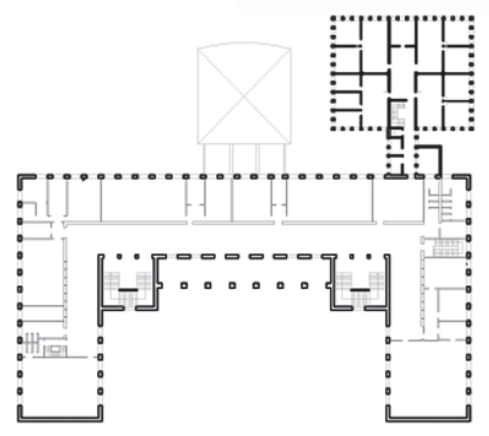

volumen paralelepipédico de cinco alturas que se situaba a un lado de la pieza en $U$ original y paralelo a la fachada trasera. Este pabellón anexo, que alojaba una biblioteca y nuevos espacios para seminarios y despachos, se conectaba con el edificio original a través de un estrecho elemento de similar altura a esta nueva pieza. Lo prosaico de la intervención, que rompía con la simetría de la composición original al tiempo que, por su mayor altura, alteraba la integridad horizontal de su alzado, quedaba soslayado por el relativo mimetismo aplicado a la construcción de sus fachadas, de materiales y diseño de huecos similares, y el respeto de la alineación de fachada del edificio de 1939.

Tras un frustrado intento de realizar una reforma interior para conseguir más espacio destinado a aulas, la intervención más importante llegaría en 1983, con la construcción del nuevo aulario. Esta ampliación daba respuesta a la imperiosa necesidad de espacio derivada del incremento en el número de alumnos y alumnas desde la creación del edificio original con once nuevas grandes aulas con capacidad simultánea para 2800 estudiantes, con una previsión de un alumnado casi paritario $^{6}$, una planta dedicada a seminarios, un cuerpo de cuatro plantas destinado a usos departamentales, entre otros usos administrativos y de servicios, y, sobre todo, una enorme dotación de espacios de relación. El nuevo aulario supondría un incremento de casi $10000 \mathrm{~m}^{2}$, frente los $6020 \mathrm{~m}^{2}$ del primero y los $2000 \mathrm{~m}^{2}$ del pabellón anexo .

\section{VENTANA SECRETA, SECRETO JARDÍN}

"Es la mejor habitación de la casa, al menos para mí, porque casi nadie va allí, salvo yo. Tiene una ventana secreta y da a un jardín secreto"8.

Una vista aérea del Campus de San Francisco ilustra de manera muy vívida hasta qué punto la ampliación de 1983 supone un momento de singularidad dentro del que hasta entonces había sido un campus particularmente homogéneo, una condición de rara avis que se mantendrá hasta hoy en día (figura 2). Para esta fecha, la universidad había continuado su crecimiento, añadiendo nuevas facultades y ampliando tanto las originales como estas últimas, pero siempre siguiendo el espíritu de los edificios ideados por Borobio y Beltrán. Volumetrías surgidas de la concatenación de paralelepípedos de directriz longitudinal, esquemas simétricos, fachadas con una disposición regular de huecos, o el uso masivo del ladrillo serían rasgos que, combinados en diferentes proporciones, garantizaban su integración en el entorno construido. Incluso otros edificios en principio discordantes, como los proyectos hermanos de los arquitectos José Luis García-Marquina y Gloria Rahola para las facultades de Matemáticas (1977-79) y

6. En el curso 1972-73, casi 7000 estudiantes de Derecho en el conjunto del país eran mujeres, lo que representaba casi un $26 \%$ del total de matriculaciones. Tres años más tarde, este número llegaría a las 16000, siendo el $34 \%$ del total. Aunque las pioneras del derecho en España pueden rastrearse hasta el último cuarto del XIX, tras la Segunda República y hasta 1966, las profesiones jurídicas -salvo la abogacía- permanecerían cerradas a las mujeres. Véase CAUSAPÉ GRACIA, Belén. La Facultad de Derecho de Zaragoza desde una perspectiva histórica de género. En: Josefa Dolores RUIZ RESA, ed. Las mujeres y las profesiones jurídicas. Madrid: Editorial Dykinson, 2020, pp. 23-41.

7. En realidad, el proyecto era aún más ambicioso, ya que contemplaba una reforma integral del edificio preexistente que permitiera a ambos funcionar como un todo. Sin embargo, la universidad decidiría finalmente abandonar esta idea. Posteriormente, entre 1992 y 1994, el arquitecto Basilio Tobías realizó una última ampliación. Esta última, más ortodoxa, aumentaría la superficie y la altura del edificio original de Borobio y Beltrán al añadir una planta más, ligeramente retranqueada. La maniobra tendría dos efectos contrapuestos: por un lado, reforzaba la presencia de la facultad en la plaza central que preside, incrementando su escala para hacerla más acorde con esta; y, por otro lado, contribuía a la imperceptibilidad del nuevo aulario, un rasgo que, se argumenta en este texto, es, en cierto modo, una de sus señas de identidad. Véase GONZÁLEZ MARTíNEZ, C.; BIEL IBÁÑNZZ, P.; HERNÁNDEZ MARTíNEZ, A., coords., op. cit. supra, nota 1. 8. KING, Stephen. Ventana secreta, secreto jardín. En: Las cuatro después de medianoche. Barcelona: Ediciones B, 1992. 
3. Planta semisótano (izquierda), planta principal (centro) y planta primera (derecha) del estado de la Facultad de Derecho de Borobio y Beltrán antes de la ampliación del nuevo aulario.

4. Fotografía aérea del nuevo aulario y del edificio antiguo de la Facultad de Derecho, Campus de San Francisco de la Universidad de Zaragoza.

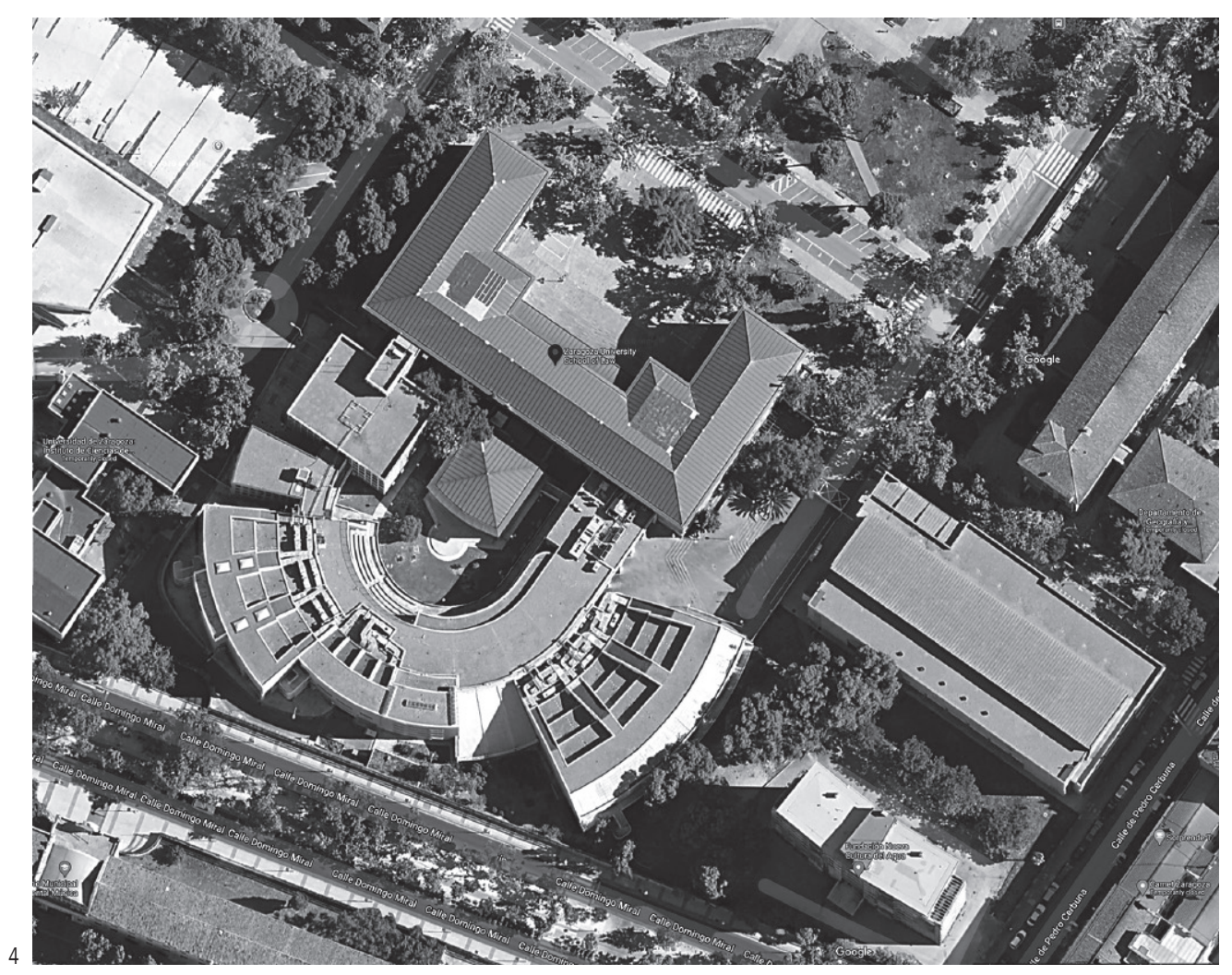

Geológicas (1978-85) ${ }^{9}$, con sus fachadas acabadas en prefabricado de hormigón, siguen, en último término, los patrones de implantación establecidos: un cuerpo lineal de disposición simétrica al que se accede a través de escaleras en el centro de su lado largo, en el primer caso, y una $L$ con lados de desigual longitud y altura en el segundo. Lo mismo puede decirse de la Facultad de Magisterio (2009-2013), última de las grandes actuaciones construidas hasta la fecha en el campus, de Javier Maya y Estela Arteche (MAAR). Pese a sus geometrías quebradas y a su materialidad de hormigón visto, esta nueva facultad no deja de ser una escultura de cuerpo redondo cuyo volumen articula el espacio a su alrededor.

Frente a todos estos, el edificio de Cisneros, Carmona, Fernández y Elorza aprovecharía su ubicación en la parcela situada detrás del primer edificio de Borobio y Navarro y limítrofe con la calle Domingo Miral para introducir un modo de implantación y un vocabulario formal diferentes de los comunes al resto de edificios y, por ende, del propio edificio que ampliaban. Pero al tiempo respondía "a un planteamiento global" que buscaba dar como resultado "una facultad completa e indivisible en su conjunto"10 (figura 4).

9. Aunque la autoría oficial de la Facultad de Matemáticas corresponde a José Luis García-Marquina, en una entrevista realizada por los autores a Gloria Rahola el 13 de abril de 2020, ella confirmó que fue coautora del proyecto. La Facultad de Geológicas ya es oficialmente obra de ambos arquitectos.

10. CISNEROS LACRUZ, Saturnino; CARMONA MATEU, Juan Antonio; FERNÁNDEZ RAMÍREZ, Manuel; GARCíA ELORZA, Isabel. Memoria del Proyecto. En: Proyecto de ejecución, ampliación y reforma de la Facultad de Derecho (Zaragoza), 1983, p. 10. 
5. Acceso al nuevo aulario. Al frente puede verse alzado lateral del volumen de aulas mayores y a la derecha el nuevo pórtico de doble altura de acceso a esta ampliación de la facultad.

6. Planta general del Proyecto de Ejecución, Ampliación y Reforma de la Facultad de Derecho (Zaragoza). Reproducción del plano original.

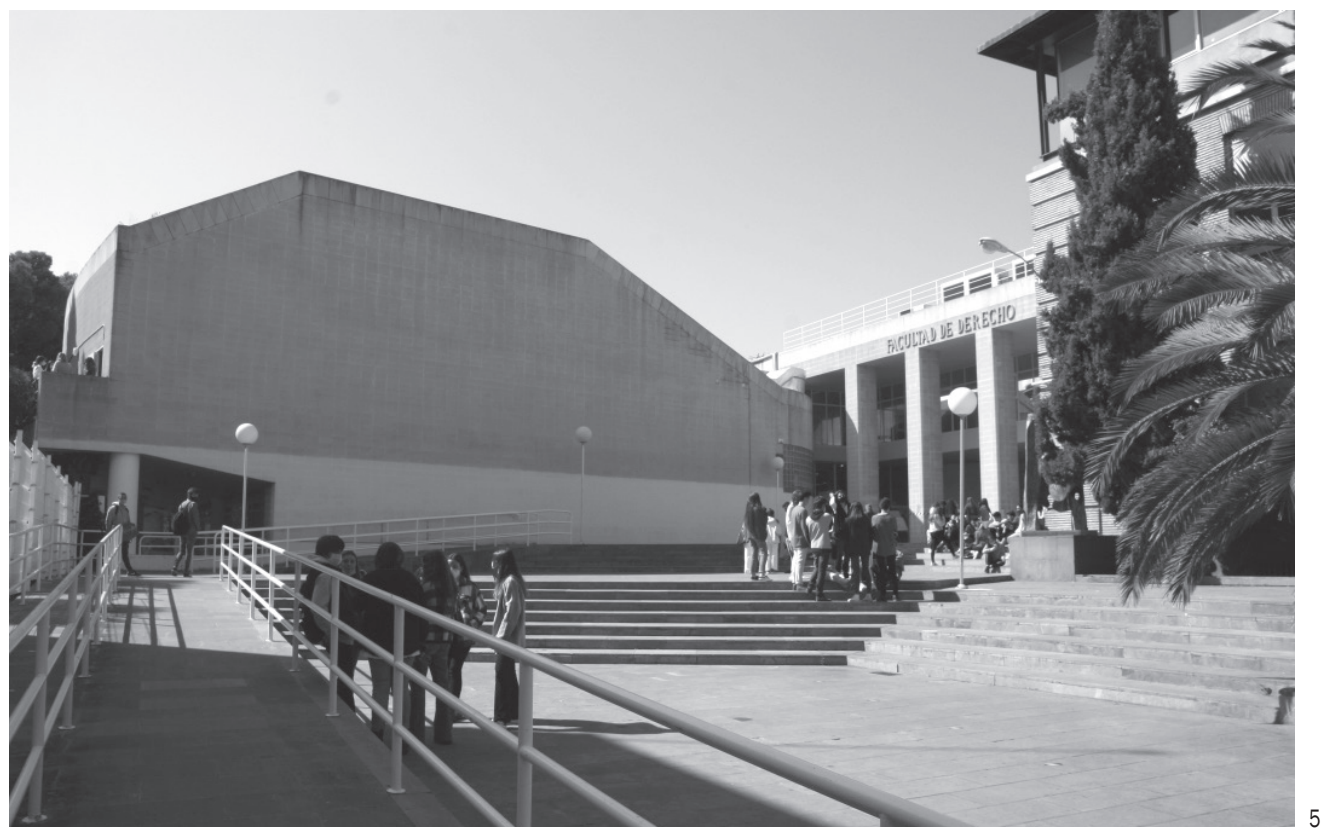

La parcela trapezoidal en la que se desarrolla el edificio era un espacio residual, con marcado carácter de trasera, que había estado ocupado ya desde los años cincuenta por unas pistas deportivas incongruentemente dispuestas en una posición diagonal, y que aparecía delimitado en sus otros dos lados por dos piezas de menor superficie. Al oeste, el reducido volumen de Instituto de Ciencias de la Educación (1963-68) constreñía la parcela y limitaba su expansión, desde una posición casi tangente al plano delimitado por la fachada lateral del edificio original. Al este, algo más lejos, la residencia de profesores de Borobio y Beltrán presentaba una aún más reducida ocupación en superficie, pero su altura de 11 plantas le confería una presencia notable.

En este contexto, los tres arquitectos y la arquitecta optaron por plantear una solución que generase un edificio volcado en sí mismo y que se diluyese en el entorno. En el proyecto original, la fachada noroeste aparecía envuelta por un enorme andamio metálico, una celosía vegetal finalmente no construida que habría ocultado aún más su presencia. Estas transparentes estructuras metálicas formalizaban la transición entre el nuevo aulario y las zonas arboladas del exterior - estas últimas tan solo serían ejecutadas en una versión muy reducida-, mientras el resto del perímetro se cercaría con una escueta verja metálica que daba al conjunto un aspecto inacabado. Las condiciones de contorno obligaban a que el acceso exterior se produjera necesariamente desde el este, donde una plaza escalonada se desparramaba por el costado del edificio preexistente, recogiendo frontalmente el flujo peatonal procedente de este. En su cota superior, esta plaza se introducía bajo la prolongación del brazo corto del primer edificio, zona de encuentro de ambos volúmenes marcada por un pórtico de doble altura que emulaba el lenguaje de la fachada principal del edificio original y tamizaba el paso hacia el jardín interno al tiempo que daba acceso al edificio (figura 5).

Por otro lado, la composición del edificio replicaba en cierto modo la forma en $U$ del original, pero partiendo, en este caso, de una única pieza de directriz circular que envolvía la cuasicúbica aula magna del primer edificio encerrándola en un jardín únicamente accesible desde el interior del edificio- y cuyos brazos se prolongaban hasta conectarse con aquel (figura 6). Este crescent asimétrico, de crujía similar a los brazos del edificio de Borobio 


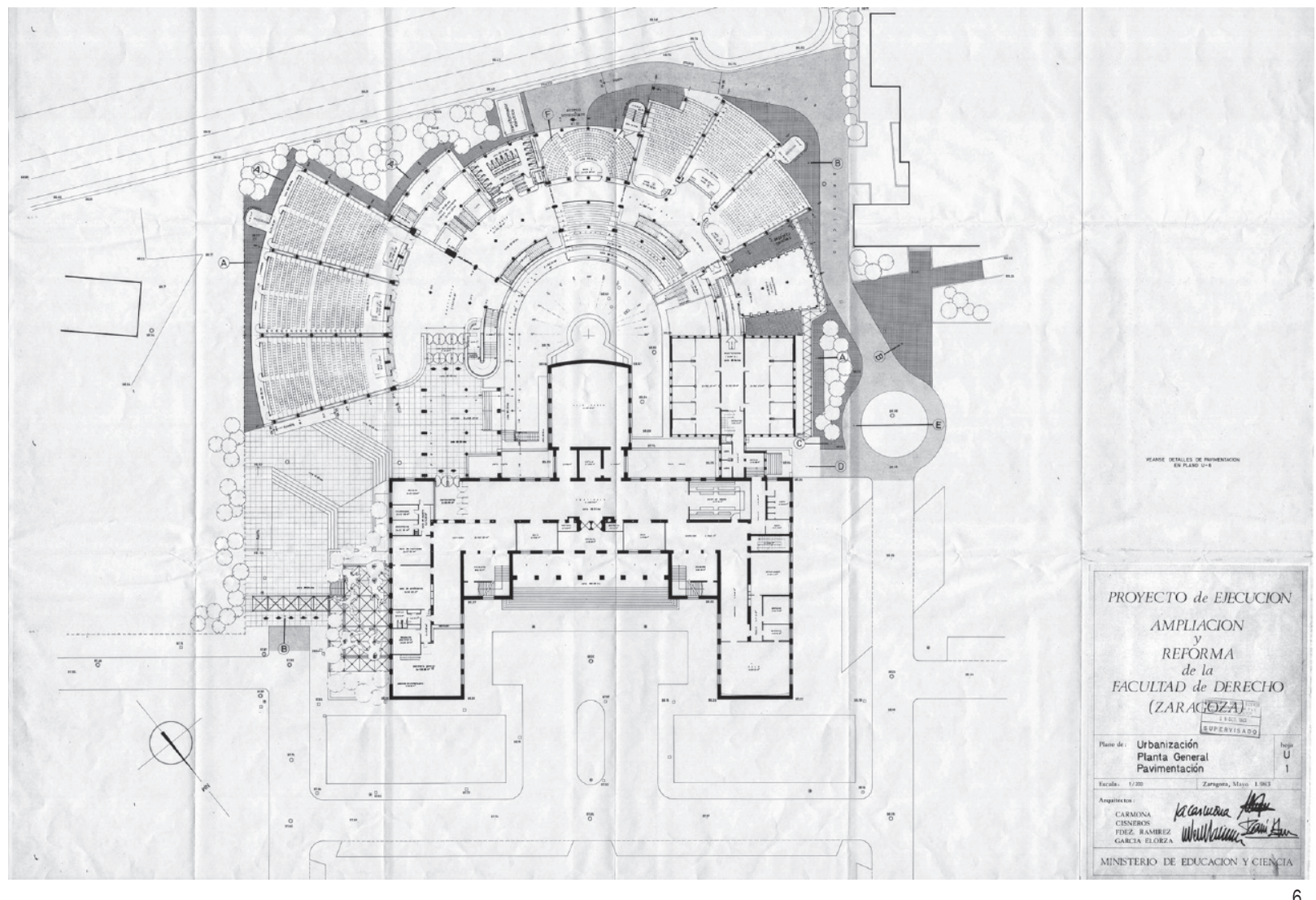

y Navarro, alojaría un complejo sistema de espacios de circulación y de socialización para estudiantes y personal docente, investigador y administrativo, tanto vertical como horizontal; un nuevo y vital espacio interior, complejo y rico en relaciones espaciales. La conexión con la facultad original se realizaba también a través de este espacio circulatorio en sus dos plantas, lo que garantizaba el buen funcionamiento del conjunto. Así, el proyecto conjuga autonomía y una marcada personalidad formal con subordinación al edificio original, tanto física como funcional. Se genera, así, una pieza cuya rotundidad volumétrica es compatible con el carácter amable para el usuario, de modo que la escala percibida desdice su gran volumen construido.

\section{CLUSTER FACULTY (CLUSTER VS. CLOISTER)}

"What we are after is something more complex... We are more concerned with 'flow' than with 'measure'. We have to create architecture [...] which, through built form, can make meaningful the change, the growth, the flow, the vitality of the community"11.
Alrededor del espacio circulatorio anterior las diferentes funciones se enchufaban, arracimándose en cuerpos con forma de sector de corona circular y profundidad y altura variables que se desarrollaban independientemente, prolongándose hasta llegar al límite permitido por el entorno. Comenzando en el este, junto al pórtico, un primer cuerpo englobaba las tres aulas mayores, para 400 personas cada una, formando el cuerpo de mayor tamaño, y determinaba en gran medida la percepción de la facultad hacia el exterior, ofreciendo su testero lateral como telón de fondo de la plaza de acceso. Tras este, un módulo de menor altura, con su cubierta de vidrio inclinada al exterior, albergaba un espacio en cascada, que generaba espacios de esparcimiento al tiempo que conectaba física y visualmente la planta de acceso con el semisótano. Tras él, dos cuerpos de mayor desarrollo repetían su programa en las plantas baja y primera (figura 7). El primero, de menor tamaño, englobaba los aseos y un aula para 200 estudiantes por planta. El segundo congregaba otras tres aulas en cada nivel. Separado de este último, un cuerpo de similar altura, pero dividido interiormente en 

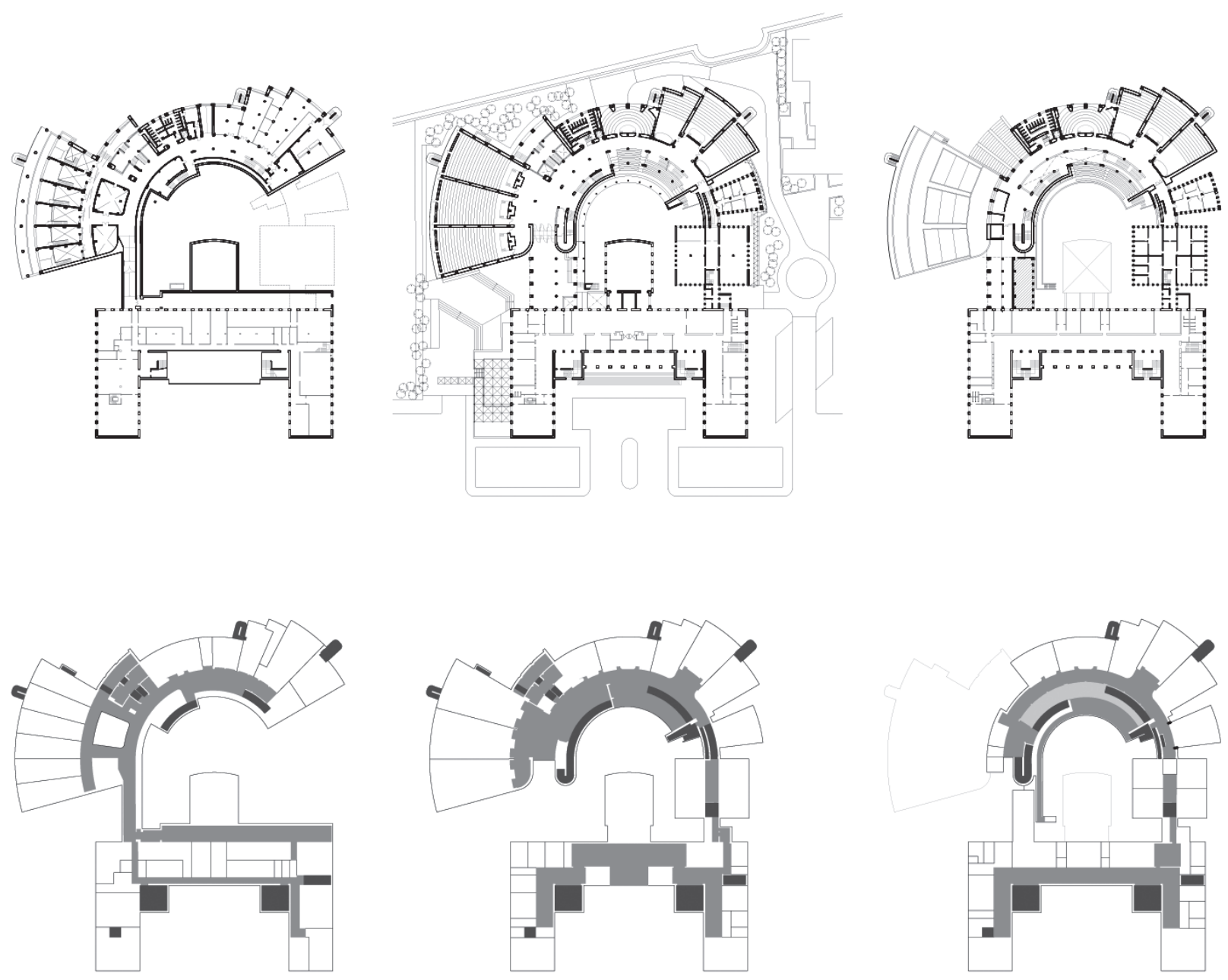

- Núcleo comunicaciones
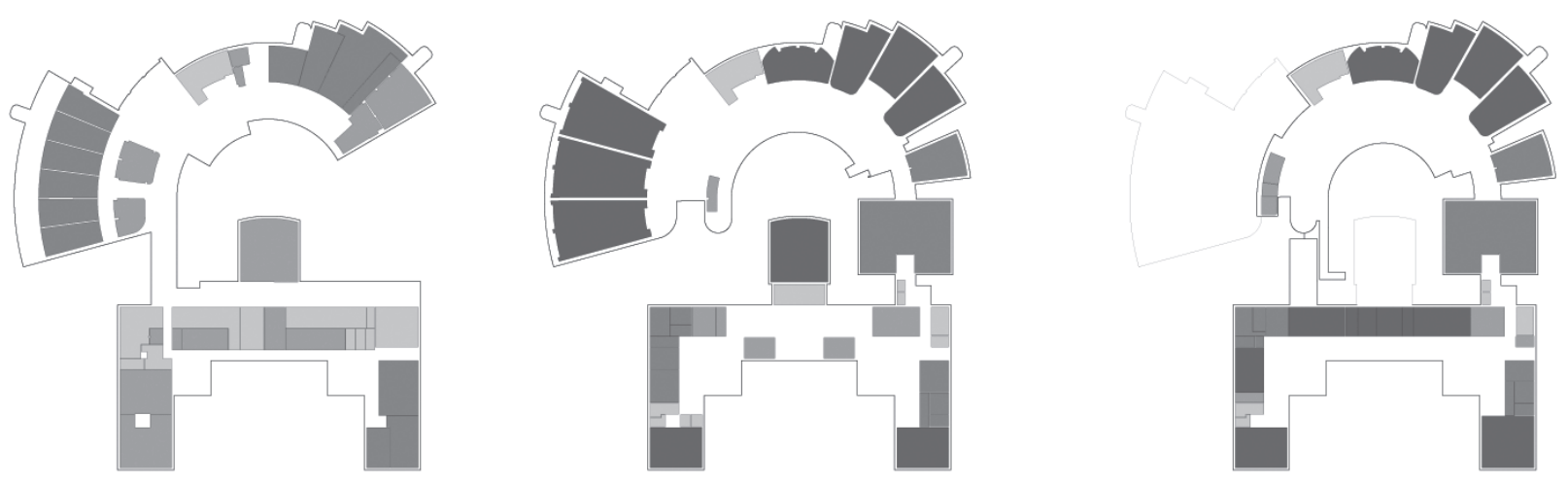

Aulas

Administrativo

Otros usos 
7. Planta semisótano (izquierda), planta principal (centro) y planta primera (derecha) del Proyecto de Ejecución, Ampliación y Reforma de la Facultad de Derecho (Zaragoza) junto con el edificio preexistente. En la primera fila puede verse la distribución general de las tres plantas del proyecto. En la segunda fila se encuentran sombreados el anillo interior con sus espacios de relación y circulación y en la fila inferior están sombreados los espacios dedicados a aulas, despachos, zonas de administración y servicios. cuatro niveles, albergaba los espacios departamentales y resolvía la transición hacia la ampliación de 1972, cuya fenestración reproducía.

Esta disposición en planta de los usos no solo responde a una lógica de mejor aprovechamiento de la parcela, sino también a criterios funcionales. Así, las aulas mayores, destinadas a los dos primeros -y masificadoscursos vertían al vestíbulo, junto al acceso, mientras el resto, destinadas al resto de cursos, se disponían más adelante, separadas ambas zonas por la sala común de esparcimiento y los aseos. Al fondo, el bloque de departamentos permite la conexión directa del profesorado tanto con el aulario como con la zona de departamentos existente. Por debajo de todos ellos, la planta de semisótano completaba el programa con una amplia superficie dedicada a seminarios y otros usos no docentes, conectando, en la zona bajo el pórtico de acceso, con el bar del edificio original y su patio inglés anexo. Visto desde el aire, el edificio aparecía como un crescent de cubierta plana cuya espalda dentada se hubiese metamorfoseado, adaptándose al irregular contorno, hasta colmatar el espacio delimitado por la parcela.

La génesis, el alma y, en último término, el éxito del proyecto, residen, en cualquier caso, en el anillo interior (figura 7). Así lo establece su memoria, que fija como objetivos arquitectónicos básicos la "[c]reación de un corazón-núcleo, alrededor del cual se polarizará la vida de la Facultad", y de "un espacio exterior propio de la Facultad de Derecho: [el] Claustro"12. Así, frente a la libre disposición del programa opaco, articulada de un modo elemental, por simple yuxtaposición de una serie de cuerpos dispuestos radialmente, el anillo interior supone un complejo y estricto mecanismo circulatorio cuya crujia se somete a una serie de dilataciones, sustracciones e invasiones. Entre las primeras se halla el área contigua al acceso, en que el anillo libre penetra en el cuerpo de aulas mayores para expandirse en un espacioso vestíbulo. Entre las sustracciones, destacan el vaciado -total en planta baja y parcial en la primera, que balconea sobre el acceso- del pórtico de entrada, operación que se repite en el interior. Aquí, un vacío vertical de directriz concéntrica recoge las escaleras, acostadas contra los paramentos que dan al patio, generando toda una serie de visiones cruzadas entre niveles. Pese a su sencillez conceptual, la forma curvada del espacio produce una multiplicación perspectiva que lo dota de una sensación espacial de una gran complejidad (figura 8).

El momento crucial en este espacio sobreviene, sin embargo, cuando excede su estricta función como elemento de comunicación para convertirse en ese activador social que prevé la memoria del proyecto. Como indicaban los autores, la fachada interior de este cuerpo, abierta hacia el jardín, adquiere la forma de un claustro con dos alturas: a la intemperie, pero cubierto, en su planta inferior, a cielo abierto en planta primera (figura 9). Ambos recogían los niveles interiores del edificio original. Sin embargo, su cota no era la de los espacios de circulación antes definidos, sino la de dos graderíos, interiores en planta baja y exteriores en la primera que, mediante la apertura de los paramentos acristalados, transformaban el jardín en un inesperado auditorio, una curia o, quizá más apropiadamente, un tribunal.

Esta voluntad centrípeta, de diseñar un edificio volcado hacia el interior, determinaría la elección de paramentos y la imagen externa del proyecto. Si los edificios del campus se habían presentado al entorno a través de homogéneas fachadas de ladrillo con un estudiado equilibrio entre muro y fenestración, el nuevo edificio polariza este sistema en dos respuestas antitéticas: hacia el claustro, buena parte de los muros se disuelve en grandes superficies acristaladas de suelo a techo (figura 9). Hacia el exterior, el edificio se desgaja en un rosario de cuerpos pétreos, levantados con un bloque de hormigón estriado que responde al contexto de una manera más amable de la que cabría esperar. Sus opacas envolventes, apenas surcadas en algunas zonas por estrechas ventanas rasgadas, construyen un paisaje escultórico hermético y abstracto que, sin embargo, la retina del observador parece tender a no registrar. Únicamente bajo la planta del acceso, donde el terreno circundante se manipula de formas diversas, el edificio se abre francamente a través de grandes ventanas que miran a su propio y secreto jardín (figura 10). 


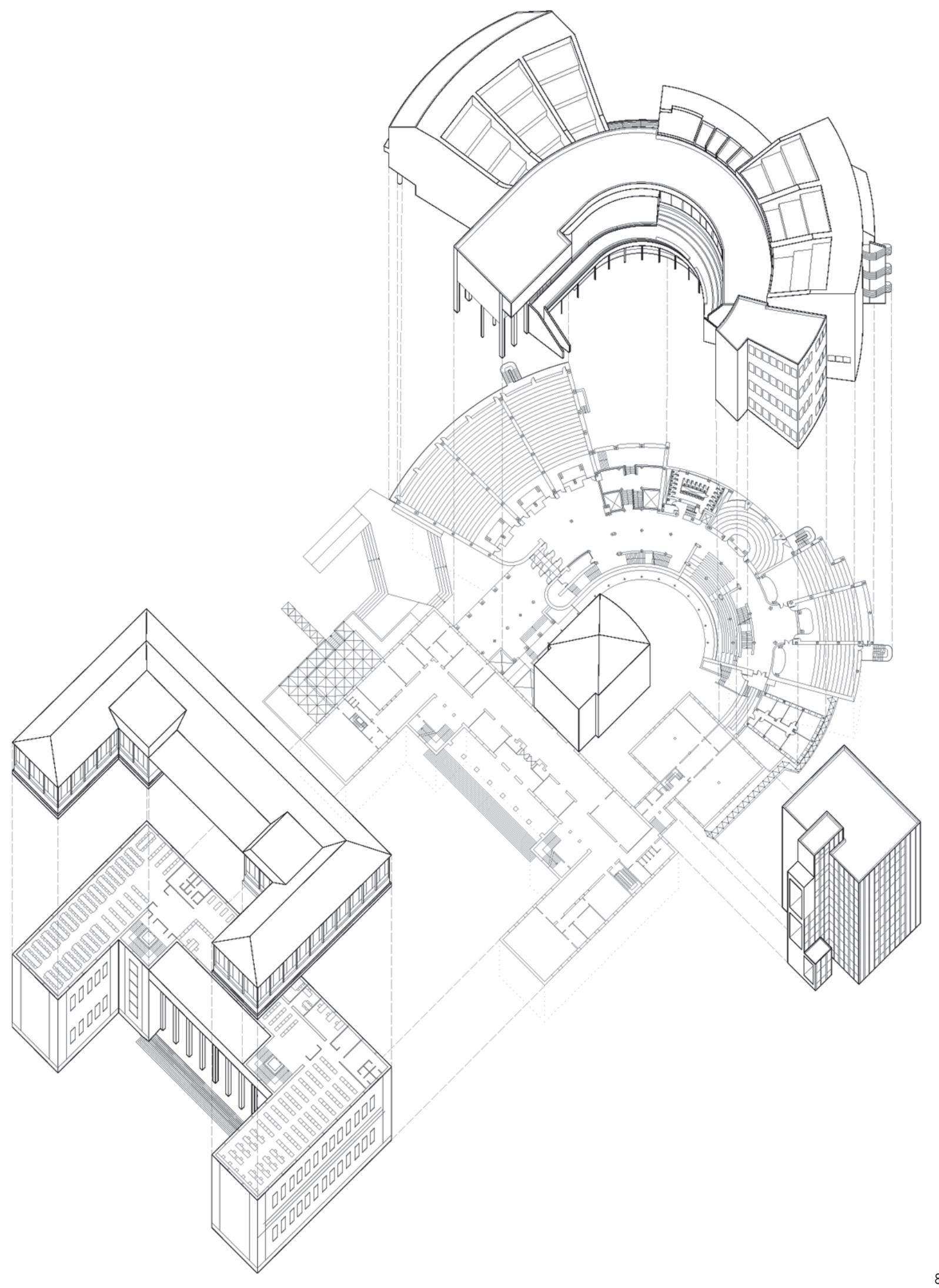


8. Axonometría que muestra cómo se relacionan los diferentes volúmenes y espacios del proyecto original y sus ampliaciones.

9. Anillo de circulación. De izquierda a derecha: graderío interior visto desde planta primera, claustro planta primera, graderío exterior.

10. De izquierda a derecha: exterior del cuerpo de espacios departamentales, exterior del cuerpo de aulas menores, $\mathrm{Ha}-\mathrm{Ha}$ en zona de seminarios bajo cuerpo de aulas mayores.
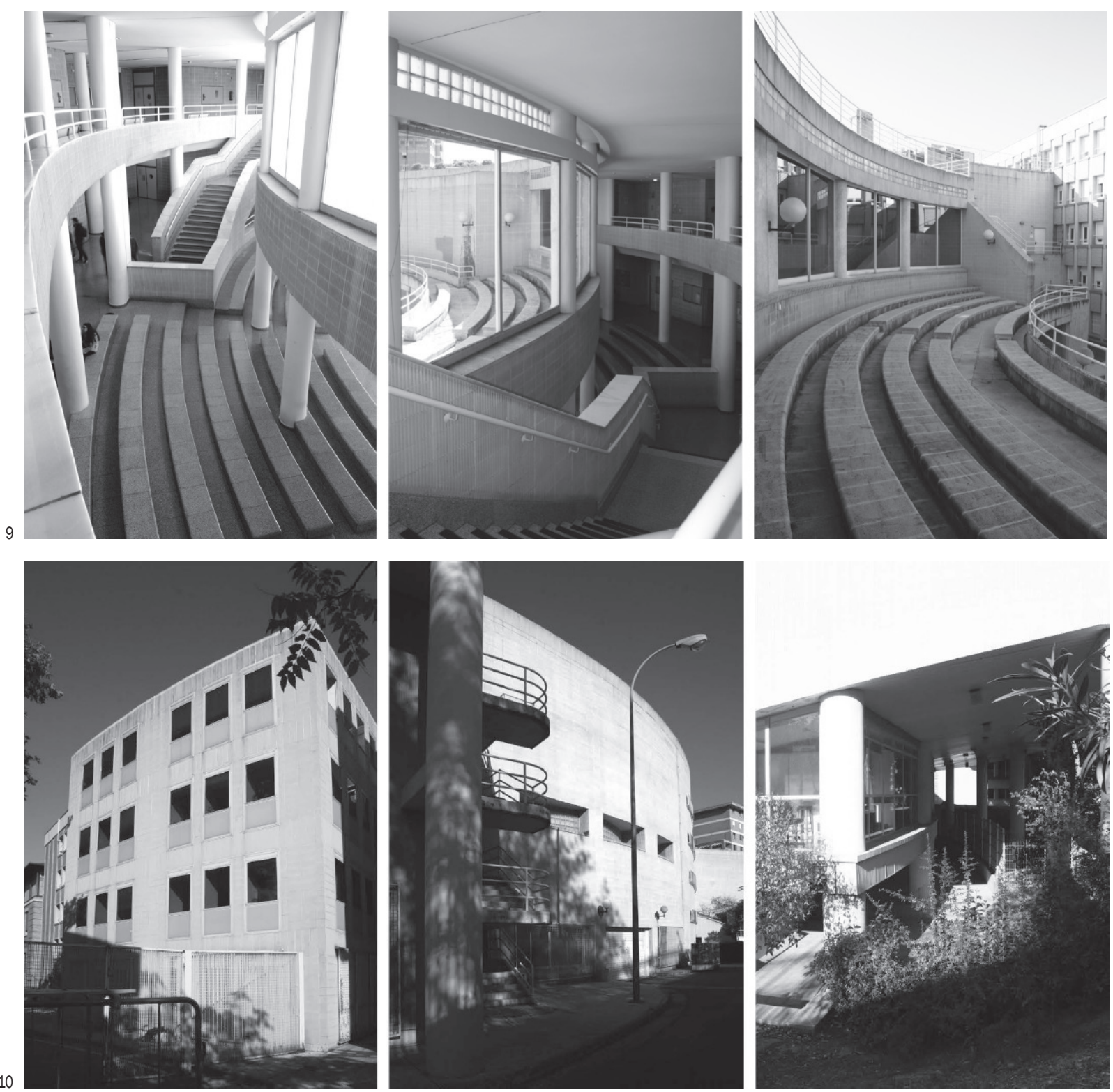

UNA OBRA EN(TRE) DOS TIEMPOS

En su revisión de la arquitectura de la Universidad de Zaragoza, Pilar Biel y Ascensión Hernández señalan que " $[\mathrm{e}] n$ la lógica sucesión de estilos y tendencias arquitectónicas producida con el paso del tiempo, al funcionalismo y al brutalismo le seguiría la aparición del postmodernismo, que también tiene su manifestación en nuestra arquitectura universitaria [con un ejemplo señalado en] la ampliación de 
11. Zona exterior del edificio donde puede verse la galería exterior de evacuación a la que tienen acceso directo el volumen de las aulas. la Facultad de Derecho"13. En la primera categoría se encuadrarían varias de las arquitecturas proyectadas durante los sesenta, mientras que la segunda es asignada por las autoras a los edificios de García-Marquina y Rahola, a la que ciertamente corresponden en un uso convencional del término. Más problemática es la última de las adscripciones. Tal vez sean los ecos de arquitecturas grecorromanas (figura 9) -"ágora" o "teatro clásico" son algunos de los términos con que se describe el proyecto- o el cierto aroma pintoresquista que el paso elevado interior, con su forma evocadora de otras pérgolas comunes al lenguaje posmoderno de la época, confiere al jardín, lo que lleva a las autoras a esta categorización. Quizá sean las citas importadas de los edificios preexistentes. Pese a su relativa autonomía formal, el edificio incorpora algunos guiños en su encuentro con aquellos: el pórtico de acceso supone, por proporción y geometría, una refacción del construido por Borobio y Beltrán en la fachada principal (figura 1 y figura 5). Y lo mismo ocurre en el nuevo cuerpo de departamentos, donde el aulario se metamorfosea y crea un doppelganger distorsionado del original, a medio camino entre el lenguaje de ambos, para favorecer la transición entre lo nuevo y lo existente. Faltan, en cualquier caso, la ironía o la voluntad metalingüística que se le suponen al juego posmoderno. Los dos elementos citados son más importaciones o préstamos que se introducen para crear una serie de rimas visuales. Son antes gestos miméticos que juegos semánticos cuya capacidad anamnésica es, en este sentido, muy limitada ${ }^{14}$.

Así, pese a que el edificio se encuadra cronológicamente en la posmodernidad, poco hay de posmodernismo en su diseño, que entronca, en realidad, mucho más directamente con una tradición netamente moderna, tanto la correspondiente a su etapa heroica como su interpretación a partir de los últimos años cincuenta. Es inevitable, por ejemplo, percibir ecos aaltianos tanto en el volumen como en la planta, que remiten a proyectos como el Wolfsburg Cultural Center en Berlín (1959-1962). Al mismo tiempo, las formas radiales, de directriz circular, completa o no, fueron esquemas compositivos habituales en la arquitectura moderna durante los años sesenta y siguientes, estando particularmente presentes en la obra de arquitectos adscritos de una u otra manera al brutalismo. Ahí están, por ejemplo, la embajada de Estados Unidos en Dublín (1959-64) de John M. Johansen, entre las circulares, o la guardería Yukari Bunka en Tokio (1967), de Kenzo Tange, que jugaba con la diferente profundidad de los sectores para adaptarse a un solar de perímetro particularmente irregular. Asimismo, comparte estrategias con intervenciones contemporáneas en conjuntos universitarios como Keble College, donde el De Breyne Building / Hayward Quad (1977), de Ahredns, Buron y Koralek, definía el perímetro por medio de una pieza curvilínea que envolvía a los edificios originales, de estilo victoriano. Con una hermética fachada de ladrillo hacia el exterior, el edificio se desmaterializaba hacia el jardín interior por medio de un cerramiento continuo de vidrio que formaba un claustro cubierto y semienterrado en la planta inferior. Tampoco resulta lejano el anterior New Court (1966-1970) en Christ's College, de Denys Lasdun, cuya hoy desaparecida -y levemente curvada- fachada original guardaba un volumen escalonado que se desparramaba hacia el espacio ajardinado en el interior de la manzana.

En el ámbito nacional, estos esquemas circulares pueden buscarse, entre otros, y de forma muy señalada, en la obra de Fernando Higueras a lo largo de los años sesenta. Son particularmente notables el proyecto para el concurso del Centro de Restauraciones Artísticas (Madrid, 1961), realizado junto a Rafael Moneo, y el proyecto finalmente construido junto a Antonio Miró, ya como Centro Nacional

13. BIEL IBÁÑEZ, María Pilar; HERNÁNDEZ MARTíNEZ, Ascensión. Arquitectura y arquitectos de la Universidad de Zaragoza, op. cit. supra, nota 1, p. 280. Más adelante, las autoras añaden: "El aulario de Derecho de los arquitectos Saturnino Cisneros Lacruz, Manuel Fernández Ramírez, Isabel García Elorza y Juan Antonio Mateu, destaca por su planta semiesférica [sic], de teatro clásico en la que se contraponen el carácter cerrado de sus muros con el carácter abierto del ágora en torno a la cual se abren las aulas y que permite un uso "lúdico' de un espacio docente".

14. ECO, Umberto. Lo posmoderno, la ironía, lo ameno. En: Apostillas a El Nombre de la Rosa y traducción de los textos latinos. Barcelona: Círculo de Lectores, 1997, pp. 55-64. A este respecto, y pese a ser un tanto anecdótica como fuente de referencia sobre el posmodernismo, nos remitimos a las reflexiones a este efecto que hace Eco en este texto, y especialmente a su comentario de que "en un mismo artista -una obra, en este caso- pueden convivir, o sucederse a corta distancia, o alternar, el momento moderno y el posmoderno", que, de dar por buena la categorización de Biel y de Hernández, adquiriría aquí un significado literal. 


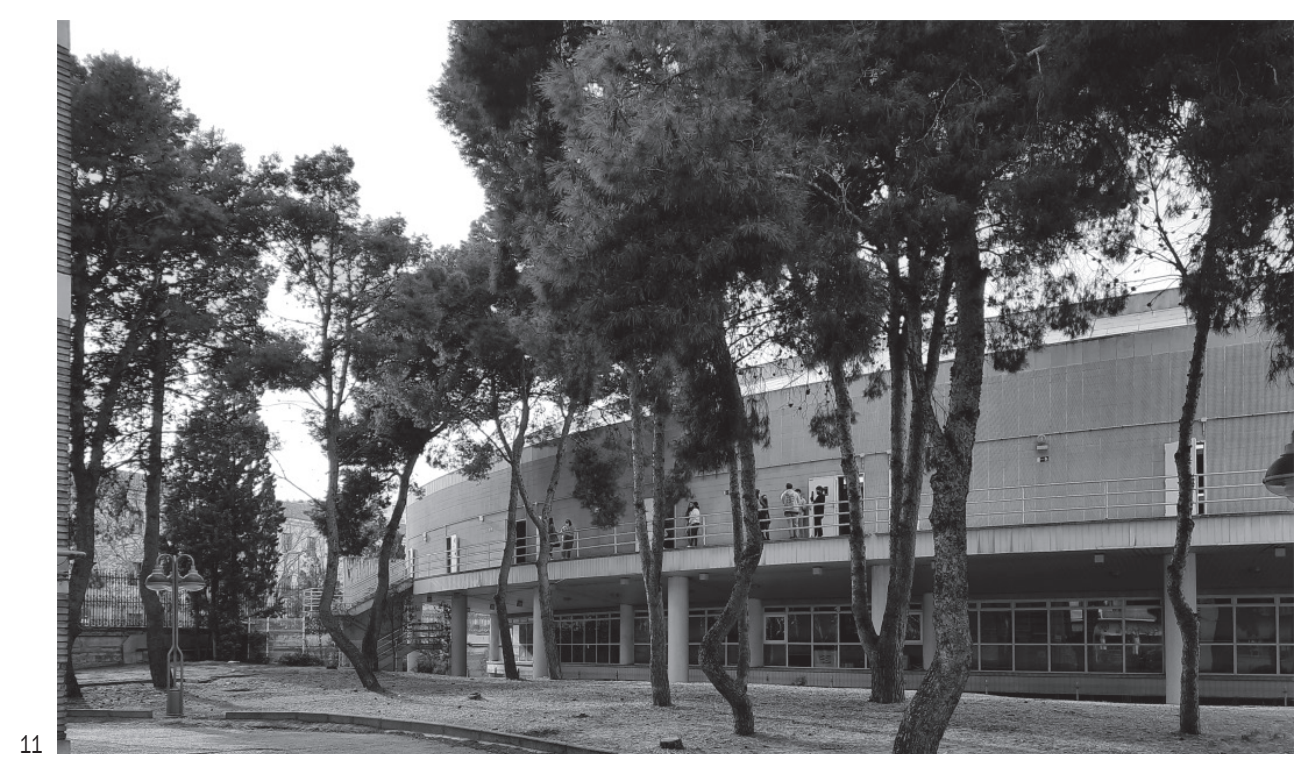

de las Artes y de la Cultura de Madrid (1967-1970), ambos con un patio circular central como corazón y elemento organizador del proyecto. También resultan relevantes propuestas como las de los concursos para el nuevo Palacio de la Ópera de Madrid (1963) o para el edificio polivalente en Montecarlo (1969), en los que el esquema circular se desarrollaba radialmente de forma centrífuga.

Sin embargo, a diferencia de todos ellos, el edificio no representa esa arquitectura muscular, caracterizada por un cierto exhibicionismo estructural aparejado al uso del hormigón que se asocia a la rama de la tardomodernidad identificada habitualmente con el brutalismo. Poco hay del ascetismo aparente -y sofisticación real- derivado del uso heroico de los materiales, y mucho de un pragmatismo nada enfático, que utiliza soluciones de, a priori, escaso pedigrí, y que, con la evidente distancia, entre otros, en la "honestidad" de la expresión material, acerca esta obra más al trabajo que Alison y Peter Smithson, notables brutalistas de diferente cuño, estaban realizando en este momento en la Universidad de Bath.

En realidad, las concomitancias con el trabajo de los Smithson pueden rastrearse más atrás aún. Es destacable, por ejemplo, el solape que se produce en la visión desde el acceso, donde el alzado lateral del volumen de aulas mayores elevado sobre pilares es flanqueado por una rampa al frente y la "calle elevada" tras él, y que de forma bastante razonable es una de las imágenes precisamente más memorables del matrimonio británico (figura 5) ${ }^{15}$ : la conocida perspectiva de su propuesta para la ampliación de la Universidad de Sheffield (1953), con su calle aérea y la no muy diferente volumetría del edificio para conferencias en primer término.

Lo mismo puede decirse de los edificios de Bath, y particularmente de sus piezas de arquitectura más prosaica, como el inacabado Arts Barn (1980-90), a los que les une no solo el uso masivo del bloque de hormigón, o el énfasis en las líneas inclinadas que introducen en los alzados las cubiertas y comunicaciones verticales exteriores, sino una determinada crudeza formal derivada de una proverbial matter-of-fact-ness en la resolución de problemas funcionales. Uno de los rasgos a priori más sorprendentes del alzado antes comentado del aulario es la aparición de un pequeño apósito en su extremo que menoscaba su rotundidad formal. La prosaica realidad es que se trata del antepecho de una galería exterior de evacuación en caso de emergencia que no solo se vuelve un rasgo particularmente protagonista de este alzado y el contiguo, sino un inesperado espacio de socialización en el cambio entre clases (figura 11).

Este es precisamente el aspecto en que el proyecto más se retrotrae al punto de origen del (nuevo) brutalismo de los Smithson como crítica de las carencias de la modernidad. Ya desde el Manifiesto de Doorn (1954), la revisión del proyecto moderno promovida por el Team $X$ había puesto el acento en la necesidad de diseñar espacios de relación que respondieran a las diferentes escalas de agrupación y en "el deseo de producir [espacios] en el que las asociaciones vitales humanas fuesen

15. En su seminal "El nuevo brutalismo", Reyner Banham establecía como una de sus características "la exigencia de que [la] forma sea aprehensible y memorable". BANHAM, Reyner. The New Brutalism. En: The Architectural Review, 1955, vol. 118, n. ${ }^{0} 708$, pp. 354-361. 

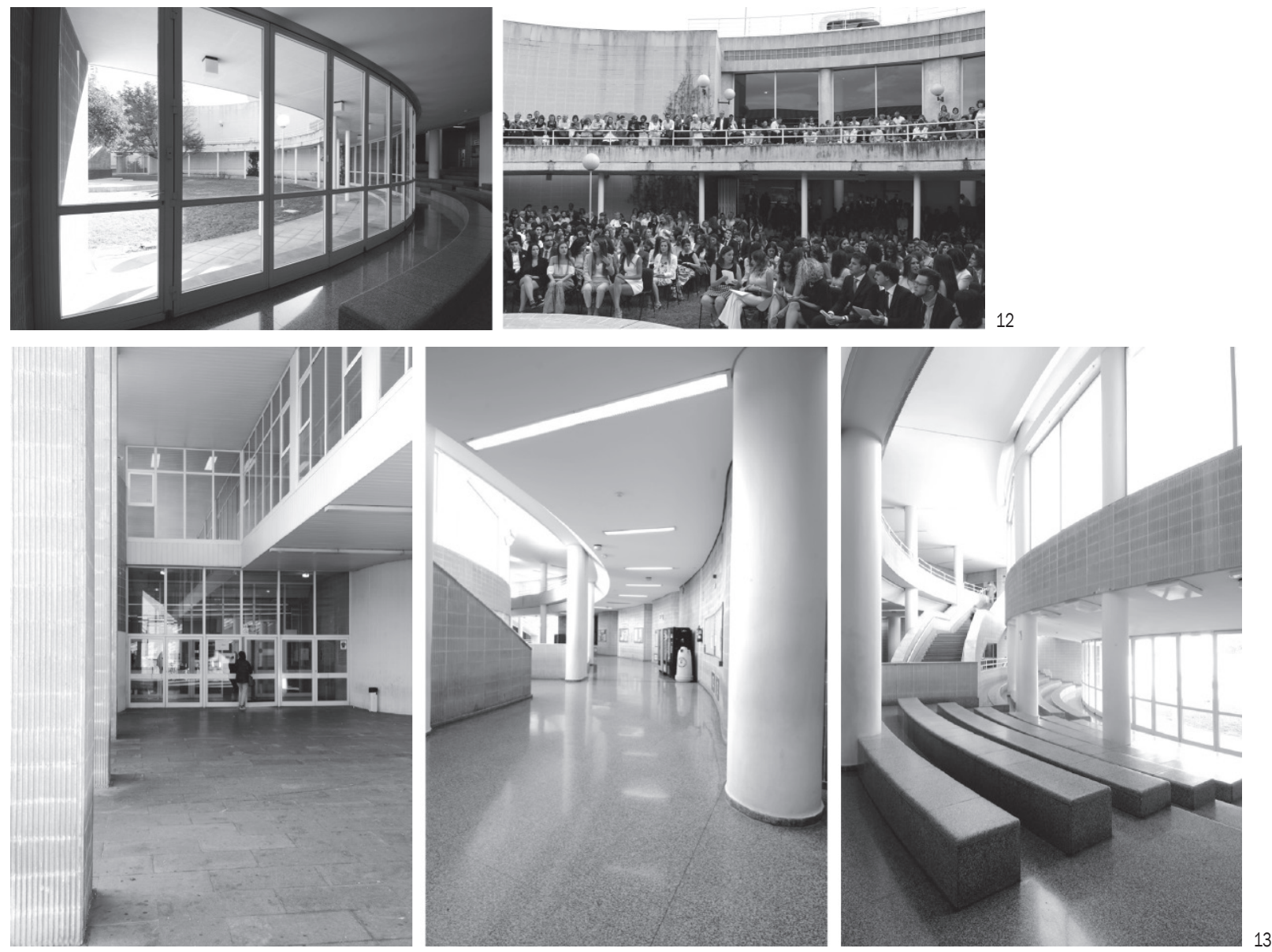

expresadas"16 en un momento en que "la estructura social a la que el urbanista ha de dar forma no es solo diferente, sino mucho más compleja de lo que había sido antes"17. El espacio de relación, como asevera la memoria del proyecto, es uno de los elementos germinales del proyecto, que en su claustro-jardín, o núcleo-corazón ${ }^{18}$ (figura 12), encontraba no solo un espacio propio e inédito en el campus, sino también un lugar de una escala acorde con el grupo de hombres y mujeres, estudiantes, docentes y personal administrativo al que sirve. Y lo hacía en un campus esencialmente construido bajo el régimen, de polarizadas relaciones interior/exterior, con límites estrictamente delineados entre la pieza construida y unos espacios comunes de vastas superficies.
El leit motiv de la ampliación de la Facultad de Derecho era la creación de un espacio comunitario en el sentido de "generador de comunidad", y esto es algo a lo que ciertamente contribuye su elaboradísimo y polimorfo espacio interior de circulación a varios niveles (figura 13). Si la intención de los Smithson era que sus galerías elevadas de comunicación se transformaran en "verdadera[s] calle[s] en el aire, [que dieran] acceso a suficientes personas para que se convierta[n] en una entidad social" 19 , este aspecto era esencial en el nuevo aulario, cuya crujía interior dejaba atrás cualquier distinción espacial entre corredor, comunicación vertical y espacio de relación, todos ellos señalados en el proyecto original con el epígrafe "estancia interclases". Como señalan Michael Kubo,

16. HEUVEL, Dirk van der; RISSELADA Max, eds. Team 10: In Search of a Utopia of the Present 1953-1981. Rotterdam: NAi Publishers, 2005, p. 43. Cita original: "[...] their desire to produce towns in which vital human associations were expressed". BANHAM, R., op. cit. supra, nota 15.

17. SMITHSON, Alison; SMITHSON, Peter. An Alternative to the Garden City Idea. En: Architectural Design, julio 1956, vol. 26, n. ${ }^{0}$ 7, pp. $229-231$.

18. Es sintomático el uso concreto de esta terminología. Las referencias al "corazón" y al "núcleo" se volverían comunes en la modernidad al menos desde la celebración del CIAM 8: The Heart of the City, como evidencia, por ejemplo, TYRWHITT, Jaquelin. Cores within the Urban Constellation. En: Congrès Internationaux d'Architecture Moderne. The Heart of the City: towards the humanization of urban life. Londres: Lund Humphries, 1951.

19. SMITHSON, Alison; SMITHSON, Peter. The Charged Void: Architecture. Nueva York: Monacelli Press, 2002. P. 86. 
12. Claustro- jardín visto desde el interior (izquierda) y desde el exterior (derecha) siendo utilizado para un acto de graduación de la facultad.

13. Espacio interior de circulación. De izquierda a derecha: acceso, corredor en planta baja y graderío interior en planta baja.

14. Espacio interior núcleo-corazón del nuevo aulario.

Chris Grimley y Mark Pasnik, el brutalismo fue "visto originalmente como un reflejo de las actitudes democráticas de una poderosa expresión cívica" ${ }^{20}$ cuya honestidad material, añade Nikil Saval, "se unió a los duros y prosaicos objetivos de la socialdemocracia".

El brutalismo es, como ha señalado Michael J. Lewis, "la expresión vernácula de/ welfare estate"21, y esto resulta aún más cierto de su encarnación original, antes de que el término adquiriera una connotación estilística. Diversos autores han señalado las raíces socialistas -o socialdemócratas-asociadas al programa del Nuevo Brutalismo22, y la democratización del bienestar que suponía su búsqueda de proveer espacios que activaran el intercambio social, la actividad ciudadana y, en definitiva, la participación en la vida comunitaria de hombres y mujeres de todos los estratos sociales. La voluntad de Cisneros, Carmona, Fernández y García Elorza de crear un núcleo que activara la vida de la facultad y, consecuentemente, el intercambio entre todos los miembros de la comunidad educativa y, sobre todo, estudiantil, ciertamente parecía apuntar en este sentido (figura 14). Y lo hacía en un momento en el que por primera vez en España se consolidaba el acceso igualitario a la universidad, tanto en lo relativo al estrato social como al género: las impactantes vistas perspectivas de los Smithson, como ya señalara Reyner Banham en "The New Brutalism", validaban "la presencia de los seres humanos como parte de la imagen total, [con] fotografías de personas pegadas sobre los dibujos de modo tal que la presencia humana casi sepultaba a la arquitectura" ${ }^{23}$. Si bien no en imagen fotográfica, las nueve secciones contenidas en el proyecto de ejecución de la ampliación de la Facultad de Derecho de la Universidad de Zaragoza mostraban una nada desdeñable población

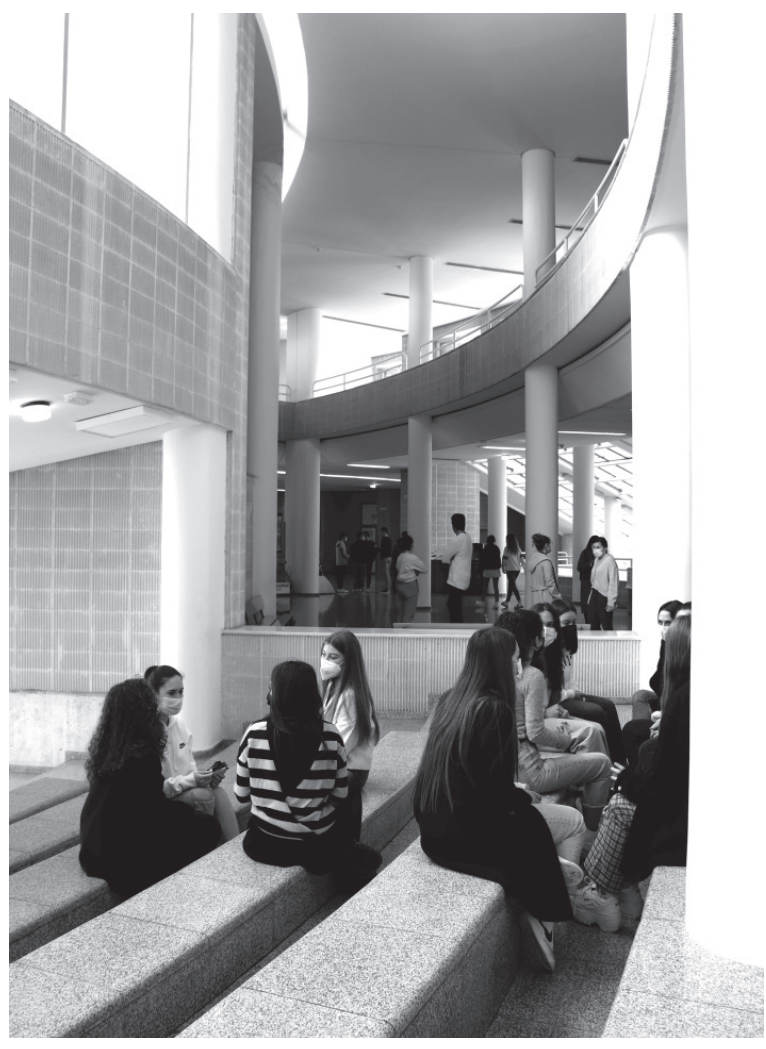

14

de 180 figuras humanas, dispuestas enfatizando los espacios de relación. Sin embargo, si en las perspectivas para su propuesta de Golden Lane Estate (1953), Alison y Peter Smithson incluían prominentemente a Terence Conran, Jawaharlal Nehru o Gerard Philipe $^{24}$, en este nuevo aulario las secciones aparecían pobladas, al fin, por un número casi igualitario de alumnos y alumnas (figura 15).

\section{UN EDIFICIO INVISIBLE, UN MUNDO INTERIOR}

Testimonio, quizá, del éxito de esta búsqueda de un mundo interior logrado sin estridencias es el hecho de que, salvo para aquellos que lo utilizan, el edificio de 1983 es, casi cuarenta años después de comenzar su construcción, un edificio que pasa desapercibido para la ciudadanía. En una discreción que contrasta tanto por su rotunda

20. KUBO, Michael; GRIMLEY, Chris; PASNIK, Mark. Brutal. En: Clog, febrero 2013, p. 166. En el texto, los autores argumentan que “la reducción del brutalismo a una etiqueta estilistica exclusivamente asociada con el hormigón coincidió con el cambio de actitudes hacia el Gobierno y el declive de la inversión estatal en el ámbito público. Originalmente visto como un reflejo de las actitudes democráticas de una poderosa expresión cívica (autenticidad, honestidad, franqueza y fuerza), la naturaleza contundente de la estética brutalista finalmente llegó a significar precisamente lo contrario: hostilidad, frialdad, inhumanidad".

21. SAVAL, Nikil. Brutalism Is Back. En: The New York Times Magazine, 2016, n. 23 , p. 53.

22. BULLOCK, Nicolas. Building the Socialist Dream or Housing the Socialist State? Design versus the Production of Housing in the 1960s. En: Mark CRISON; Claire ZIMMERMAN, eds. Neo-Avant Garde and Postmodern: Post-war Architecture in Britain and Beyond. New Haven: Yale University Press, 2010, pp. $321-342$. 23. SMITHSON, A.; SMITHSON, P., op. cit. supra, nota 17.

24. CAPDEVILA CASTELLANOS, Iván; IBORRA PALLARÉS, Vicente. Drawing a New Architectural Paradigm. En: Carlos L. MARCOS, ed. Graphic Imprints: The Influence of Representation and Ideation Tools in Architecture. Nueva York: Springer, 2019, pp. 274-284. D0I: 10.1007/978-3-319-93749-6_23 


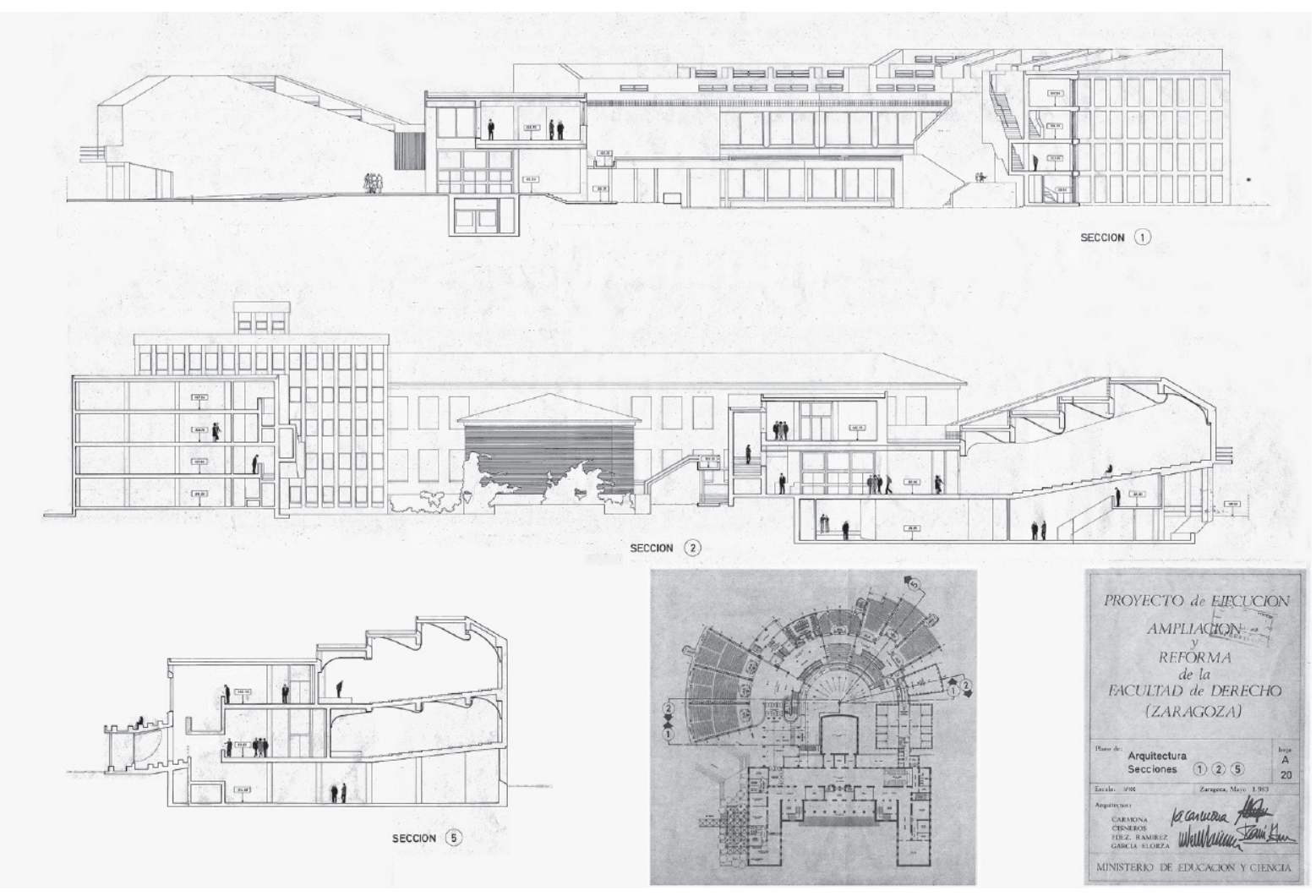

15

volumetría como por su abultada superficie, el nuevo aulario de la Facultad de Derecho es un edificio que se aparece al peatón únicamente en retazos, en visiones oblicuas y fugaces captadas mientras se desplaza entre otros puntos del campus y que son olvidadas inmediatamente; un edificio que, además, solo existe en su magnitud real en su vista aérea (figura 4). A esta cuestión contribuye tanto su ubicación, encajado entre otros edificios, preexistentes y posteriores, que han eliminado cualquier posibilidad de percibirlo en algo parecido a una visión de conjunto, como el nada favorecedor tratamiento de su entorno. Quizá, en último término, esta invisibilidad del edificio, tanto buscada como sobrevenida, no haya hecho sino garantizar su pervivencia como un edificio capaz de crear un mundo interior diferente al del resto de los primeros edificios del Campus de San Francisco: un lugar que conjuga los grandes gestos con lo cotidiano y que permanece como una cápsula del tiempo tanto del momento en que fue realizado como de las ambiciones, acaso excesivamente optimistas, de otras épocas de la modernidad. $\square$

Financiación: Este artículo es resultado del proyecto de investigación “Mujeres en la cultura arquitectónica (pos)moderna española, 1965-2000", con código PGC2018-095905-A-100, y financiado por el Ministerio de Ciencia e Innovación del Gobierno de España y los Fodos Feder de la Unión Europea. Agradecimientos: Agradecemos a la Unidad Técnica de Construcciones y Energía de la Universidad de Zaragoza su ayuda, facilitándonos para su consulta la documentación original del proyecto, y a D. Javier López Sánchez, decano de la Facultad de Derecho de la Universidad de Zaragoza, su contribución fotográfica para completar la documentación del estado actual del edificio.

Aportación de cada autor:

Luis Miguel Lus-Arana (LML-A): análisis compositivo de la obra, contextualización internacional y estructura del artículo. Coordinación redibujado y análisis gráfico

Lucía C. Pérez-Moreno (LCP-M): obtención y edición de la documentación original y fotográfica, contextualización nacional. Coordinación edición final. Autoría del artículo LML-A y LCP-M (50\% - 50\%) 


\section{Bibliografía citada}

BANHAM, R. The New Brutalism. En: The Architectural Review, 1955, vol. 118, n. 708 , pp. 354-361.

BIEL IBÁÑ̃EZ, María Pilar. Autarquía y arquitectura industrial en Zaragoza. En: Artigrama, 2003, nº. 18, pp. 527-548.

BULLOCK, Nicolas. Building the Socialist Dream or Housing the Socialist State? Design versus the Production of Housing in the 1960s. En: CRISON, Mark; ZIMMERMAN, Claire (ed.), Neo-Avant Garde and Postmodern: Post-war Architecture in Britain and Beyond. New Haven: Yale University Press, 2010, pp. 321-342.

CAPDEVILA CASTELLANOS, Iván; IBORRA PALLARÉS, Vicente. Drawing a New Architectural Paradigm. En: Carlos L. MARCOS, ed. Graphic Imprints: The Influence of Representation and Ideation Tools in Architecture. Nueva York: Springer, 2019, pp. 274-284. D0l: 10.1007/978-3-319-93749-6_23 CAUSAPÉ GRACIA, Belén. La Facultad de Derecho de Zaragoza desde una perspectiva histórica de género. En: Josefa Dolores RUIZ RESA, ed. Las mujeres y las profesiones jurídicas. Madrid: Editorial Dykinson, 2020, pp. 23-41.

CISNEROS LACRUZ, Saturnino; CARMONA MATEU, Juan Antonio; FERNÁNDEZ RAMÍREZ, Manuel; GARCÍA ELORZA, Isabel. Memoria del Proyecto de Ejecución, Ampliación y Reforma de la Facultad de Derecho (Zaragoza). Zaragoza: Universidad de Zaragoza, 1983.

DIÉGUEZ, Sofía. Arquitectura y urbanismo durante la autarquía. En: Antonio BONET CORREA (coord.). Arte del franquismo. Madrid: Cátedra, 1981, pp. 47-76.

ECO, Umberto. Lo posmoderno, lo irónico, lo ameno. En: Apostillas a El Nombre de la Rosa y traducción de los textos latinos. Barcelona: Círculo de Lectores, 1997, pp. 55-64.

GONZÁLEZ MARTÍNEZ, Carlos; BIEL IBÁÑEZ, Pilar; HERNÁNDEZ MARTíNEZ, Ascensión, coords. La Universidad de Zaragoza: arquitectura y ciudad. Zaragoza: Universidad de Zaragoza, 2008.

HEUVEL, Dirk van der; RISSELADA Max, eds. Team 10: In Search of a Utopia of the Present 1953-1981. Rotterdam: NAi Publishers, 2005.

KING, Stephen. Ventana secreta, secreto jardín. En: Las cuatro después de medianoche. Barcelona: Ediciones B, 1992.

KUBO, Michael; GRIMLEY, Chris; PASNIK, Mark. Brutal. En: Clog, febrero 2013, p. 166.

SAVAL, Nikil. Brutalism Is Back. En: The New York Times Magazine, 2016, n² 23, p. 53.

SMITHSON, Alison; SMITHSON, Peter, An Alternative to the Garden City Idea. En: Architectural Design, julio 1956, vol. 26, n. ${ }^{0}$ 7, pp. $229-231$.

SMITHSON, Alison; SMITHSON, Peter. Cluster City: A New Shape for the Community. En: The Architectural Review, 1957, vol. 122, n. ${ }^{\circ} 730$, pp. $333-336$. SMITHSON, Alison; SMITHSON, Peter. The Charged Void: Architecture. Nueva York: Monacelli Press, 2002.

TYRWHITT, Jaquelin. Cores within the Urban Constellation. En: Congrès Internationaux d'Architecture Moderne. The Heart of the City: towards the humanization of urban life. Londres: Lund Humphries, 1951.

VÁZQUEZ ASTORGA, Mónica. José Borobio. Su aportación a la arquitectura moderna. Zaragoza: Delegación del Gobierno en Aragón. Caja de Ahorros de la Inmaculada, 2007.

Luis Miguel Lus Arana (Portugalete, 1976) es arquitecto urbanista por la Universidad de Navarra (ETSAUN, 2001), Master in Design Studies (Harvard GSD, 2008) y doctor arquitecto (ETSAUN, 2013), realizando estudios complementarios en el Istituto di Architettura di Venezia (2000), y estancias docentes y de investigación en la Universidad Nacional de San Juan (Argentina, 2001) o la Harvard Graduate School of Arts and Sciences (2008-2009). Ha sido becario de Obra Social La Caixa (2006-2008) y Fundación Caja Madrid (2008 y 2009), y su obra ha sido expuesta en Barcelona, Cambridge, Nápoles, Chicago o Londres. Desde 2012 es profesor de Composición Arquitectónica en la Universidad de Zaragoza. Actualmente es miembro del equipo del proyecto de I+D 'Mujeres en la cultura arquitectónica española (pos)moderna, 1965-2000".

Lucía C. Pérez-Moreno (Zaragoza, 1979) es Doctora en Arquitectura por la ETSAM (2013), profesora Titular de Composición Arquitectónica en la Universidad de Zaragoza e investigadora de su Instituto de Patrimonio y Humanidades (IPH). Es arquitecta por la Universidad de Navarra (2003) y cuenta con estudios de posgrado por la Aalto University (Finlandia, 2004) y de máster por la GSAPP de Columbia University (EEUU, 2008). Está especializada en arquitectura y pensamiento feminista. Desde 2017, coordina los encuentros científicos anuales sobre 'Perspectivas de género en la arquitectura' y, es la investigadora principal del proyecto 'Mujeres en la cultura arquitectónica (pos)moderna española, 1965-2000' financiado por Gobierno de España (http://muwo.unizar.es). Además es miembro de la Asociación de Mujeres Investigadoras y Tecnólogas (AMIT), de la Asociación Española de Críticos de Arte (AECA), de la Plataforma de Mujeres en el Arte Contemporáneo (PMAC) y de la European Architectural History Network (EAHN), de cuya revista científica (Architectural Histories) ha sido editora entre 2017 y 2020. 


\section{Autor imagen y fuente bibliográfica de procedencia}

Información facilitada por los autores de los artículos:

página 19, 1. WOODBRIDGE, Kenneth. Princely Gardens. Nueva York: Rizzoli, 1986; página 21, 2. ALPHAND, J. C. Adolphe. Les Promenades de Paris. Princeton: Princeton Architectural Press, 1984, p. 199; página 22, 3. AURICOSTE, Isabelle; TONKA, Hubert. Parc Ville Villette. Seyssel: Champ Vallon, 1987; páginas 23,24 y 25, 4, 5 y 6. Fotografías de Ramón R. Llera; páginas 26 y 27, 7 y 8 . BASSO PERESSUT, Luca; CALIARI, Pier Federico, eds. Piranesi Prix de Rome. Progetti per la nuova Via dei Fori Imperiali. Roma: Aión Edizioni, 2017; página 28, 9. LAB/PAP Laboratorio de Paisaje Arquitectónico, Patrimonial y Cultural; páginas 28 y 29, 10, 11 y 12 . BASSO PERESSUT, Luca; CALIARI, Pier Federico, eds. Piranesi Prix de Rome. Progetti per la nuova Via dei Fori Imperiali. Roma: Aión Edizioni, 2017; páginas 30 y 31 ,13, 14 y 15. ÁLVAREZ, Darío; IGLESIA, Miguel Ángel de la. Modelos de paisajes patrimoniales. Estrategias de protección e intervención arquitectónica. Valladolid: LAB/PAP y Departamento de Teoría de la Arquitectura y Proyectos Arquitectónicos, Universidad de Valladolid, 2017; página 36, 1. Philippe BOURDIN, “Le sacre de Louis XV", Histoire par l'image [en línea], consultado el 20 de agosto de 2020. Disponible en: http://histoire-image.org/fr/etudes/sacre-louis-xv; página 37, 2. Archivo de José lgnacio Linazasoro Rodríguez; página 38, 3. JAKOVUEVIC, Nada; CULOT, Maurice, dirs. Places et monuments. Bruselas: Mardaga, 1984; página 40, 4. RIGAUD, Olivier. Les plans anciens de Reims, 16001825. Dossier iconographique. Reims: RHA, 1991; página 41, 5. Biblioteca Municipal de Reims [en línea], consultado el 20 de agosto de 2020. Disponible en: https:// commons.wikimedia.org/wiki/File:Rue_Libergier_Cathédrale_Reims_Varin_30131.jpg; página 42, 6. Revista UR, 1985, n. ${ }^{\circ}$ 2; página 43, 44, 46, 47, 48, 49, 50, 7, 8, 9 , 10, 11, 12, 13, 14, 15. Archivo de José Ignacio Linazasoro Rodríguez; página 54, 1. Eisenman Architects Archives; página 55, 2. DR2004:0360:003 Cedric Price fonds Canadian Centre for Architecture; página 56, 3. DR2004:0355:007. Cedric Price fonds Canadian Centre for Architecture; página 57, 4. DR2004:0355:006. Cedric Price fonds. Canadian Centre for Architecture; página 59, 5. ARCH267828. IFCCA Prize Competition for the Design of Cities fonds Canadian Centre for Architecture; página 59, 6. Dominio público.; página 60, 7. Archivo Lacaton \& Vassal; página 61, 8. Fotomontaje de los autores a partir de Google Street View (2018); página 62, 9. Fotografía de Philippe Ruault:; página 63, 10. Muestra de la web del proyecto con el reportaje fotográfico de Philippe Ruault.; página 64,11 . Archivo Lacaton \& Vassal.; página 65,12 . Vista aérea en Google Maps (2018):; página 65, 13. Vista obtenida en Google Street View (2018); página 66, 14. Archivo Lacaton \& Vassal, 2004.; página 67, 15. Vista obtenida a través de Google Street View (2018); páginas 74 y 75, 1, 2 y 3. @ Krijn de Koning, cortesía del artista; páginas 76 y 77, 4, 5 y 6 . @ Jessica Stockholder, cortesía de la artista y de Mitchell-Innes \& Nash, Nueva York; páginas ,78 y 79, 7, 8 y 9. @ Katharina Grosse, VEGAP, Sevilla, 2021; páginas 82 y 83, 10 , 11 y 12. Heather Day website. Disponible en: https://heatherday.com/downtownoakland; página 90, 1. Universidad de Zaragoza. Unidad Técnica de Construcciones.; página 91, 2. Planimetría redibujada por Clara Dobón Ricarte.; página 92, 3. Planos, redibujada por Clara Dobón Ricarte. Fotografías cortesía de la Facultad de Derecho de la Universidad de Zaragoza.; página 93, 4. Google Maps.; página 94, 5. Fotografías de los autores.; página 95, 6. Proyecto de Ejecución, Ampliación y Reforma de la Facultad de Derecho (Zaragoza). Fuente: Universidad de Zaragoza. Unidad Técnica de Construcciones.; páginas 96 y 98, 7, 8. Planos y axonometría dibujados por Clara Dobón Ricarte; páginas 99 y 101, 9, 10, 11. Fotografías de los autores.; página 102, 12. Fotografía (izquierda) de los autores y fotografía (derecha) cortesía de la Facultad de Derecho de la Universidad de Zaragoza.; página 102, 13. Fotografías de los autores.; página 103, 14. Fotografía cortesía de Javier López Sánchez, decano de la Facultad de Derecho de la Universidad de Zaragoza.; página 104, 15. Proyecto de Ejecución, Ampliación y Reforma de la Facultad de Derecho (Zaragoza). Fuente: Universidad de Zaragoza. Unidad Técnica de Construcciones; página 108, 1. Dibujos del autor; página 109, 2. Ayuntamiento de Madrid; página 110 y 112, 3 y 4. Ayuntamiento de Madrid; página 113 y 114, 5 y 6 . Archivo Cabrero; página 115, 7. Reconstitución gráfica del autor; página 116, 8. Legado 2 Fundación COAM; página 117, 9. Dibujos del autor; página 118, 10. Arquitectura, 1959, n. 7 y Francisco de Asís Cabrero y Jaime Ruiz; página 119, 11. Archivo Cabrero; página 120, 12. Dibujos del autor; página 120, 13. Fotografía del autor; página 121, 14. Dibujos del autor; página 122, 15. Fotografía del autor. 
UN EDIFICIO INVISIBLE. NUEVO AULARIO DE LA FACULTAD DE DERECHO DE LA UNIVERSIDAD DE ZARAGOZA (1983-1996)

\section{AN INVISIBLE BUILDING. NEW LECTURE ROOM BUILDING OF THE FACULTY OF LAW OF THE UNIVERSITY} OF ZARAGOZA (1983-1996)

Luis Miguel Lus-Arana (https://orcid.org/0000-0001-5826-2642)

Lucía Carmen Pérez-Moreno (https://orcid.org/0000-0002-6303-1950)

p.89 In 1983, the University of Zaragoza began the construction of an extension to its Faculty of Law'. This project would be the last of a series of new buildings constructed in the Ciudad Universitaria -currently the San Francisco Campusbetween 1969 and 1989. The pressing need for this extension stemmed from the important changes in education that had been taking place in Spain since the late Franco era, which the transition to democracy was beginning to consolidate. Ever since the 1970s, the number of people who wanted to train as lawyers had increased causing a significant and sustained rise in the number of law students, including a noticeable and unprecedented progression in the presence of females in the classrooms. In the case of the Faculty of Law in Zaragoza, this generalised increase was reflected in the need to build a new lecture room building to accommodate some 2,800 new students.

The project for the new lecture room building is the work of a team of architects formed by Saturnino Cisneros Lacruz, Juan Antonio Carmona Mateu, Manuel Fernández Ramírez and Isabel García Elorza - one of the first female architects to practice in the autonomous community of Aragón². Their design for the new lecture room building shows a respectful break from the blend of modernity and regionalism typical of the autarchic phase of Franco's regime and predominant in the architecture of the Ciudad Universitaria during the period. In contrast to both the solemn monumental nature of the buildings of the 1930s and 1940s and the sculptural play of the parallelepiped compositions of later works, the building is the result of a balance between compositional autonomy and the search for functional integration between the new and the existing. If in the original buildings the exterior presence had been fundamental in the urban definition of the campus, in the new lecture room building the creation of an interior world took precedence:

p.90 a set of architectural spaces without hierarchies, where the spaces of vital relations between students, teaching staff and administration personnel became the main consideration.

\section{THE FACULTY OF LAW AND THE CIUDAD UNIVERSITARIA OF ZARAGOZA}

The original Law Faculty building (figure 1) had been built and altered between 1939 and 1945 by Regino Borobio and José Beltrán Navarro ${ }^{3}$, six years after winning the competitive tender for preliminary projects for the development of the Ciudad Universitaria of Zaragoza (figure 2). This would actually be the second building constructed by the two men on the campus: in 1935 they had designed the Faculty of Philosophy and Letters, and between 1954 and 1966 they completed the central square of the University City with the Faculty of Sciences. The Faculty of Law would be the main component of a C shape opening towards the northeast that would eventually be closed in 1973, with the completion of the Interfaculty building and the Pavilion annex to the Isidoro Garasa Rectory (1970-73) that would mark the initiation of a second great expansion of the campus at the end of the 1960s, continuing on through the 1970s.

Law and its two neighbouring faculties, together with other buildings constructed by Borobio and Beltrán Navarro, such as the Colegio Mayor Universitario Pedro Cerbuna (1942-1950) and the teachers' residence (1964-69), located on the plot adjacent to the new lecture room building, would establish the parameters that would define the architecture of the Ciudad Universitaria. The campus buildings are generally characterised by a modern rationalism inherited from Beaux Arts compositions, with floorplans based on a Cartesian grid formed out of a concatenation of rectangular elements. The exterior appearance is marked by the severity of the elevations with regular openings and the massive use of brick in the exterior walls. In combination with the piched roofs of the first buildings, both reflect typical features of the architecture of Spain's autarchy in the $1940 \mathrm{~s}^{4}$ that, in Zaragoza, express Aragonese building traditions and the internal history of the University, associated at the turn of the 20th century with the work of the architect Ricardo Magdalena and his recovery of the architecture of the Aragonese Renaissance ${ }^{5}$.

p.91 The first building of the Faculty of Law adopted a U-shaped layout open to the large landscaped central square facing the Faculty of Philosophy and Letters to the east, the Science Building to the west and the Interfaculty building to the north. The building is organised symmetrically, with the main entrance located in the middle of its long side, marking the axis of symmetry, and accessed via a large staircase typical of Beaux-Arts architecture (Figure 1). The different interior spaces are organised in a linear fashion along the $U$-shaped block and are accessible through a wide and uniform corridor space that acts both as an entrance hall on the ground floor and as a meeting and connecting space to the classrooms and administration spaces on the basement, main, and first floors (figure 3).

To this rigid U-shaped scheme, an additional structure was added to the south, the aula magna, an almost cubic volume located on the axis of symmetry facing the great main staircase and the central hall space. This configuration would remain unchanged until October 1970, when Borobio and Beltrán designed the first of the extensions to the

p.92 faculty, a new five-storey parallelepiped volume located to one side of the original U-shaped composition and parallel to the rear façade. This pavilion annex housing a library and new seminar and office spaces was connected to the original building via a narrow element of similar height to this new piece. The prosaic nature of the addition, which broke with the symmetry of the original composition and marred the horizontal integrity of its elevation, was mitigated by the relative mimesis applied to the construction of its façades, which used similar materials and design of openings, and followed the façade alignment of the 1939 building. 
After a frustrated attempt to carry out alterations to the interior to achieve more classroom space, the most important intervention would come in 1983, with the building of the new lecture room building. This extension responded to the urgent requirement for more space to accommodate the increase in student numbers since the creation of the original building with eleven new large lecture rooms with a capacity when fully used for 2800 students, with an equal number of male and female students expected ${ }^{6}$, a floor dedicated to seminars, a four-storey section intended for departmental and other administrative and services uses, and in particular, a generous provision of spaces for interaction. The new lecture room building would add almost $10,000 \mathrm{~m}^{2}$, compared with the $6,020 \mathrm{~m}^{2}$ of the first building and the $2,000 \mathrm{~m}^{2}$ of the pavilion annex?.

\section{SECRET WINDOW, SECRET GARDEN}

"It is the best room in the house, at least for me, because hardly anybody ever goes there, except me. It has a secret window and looks down on a secret garden"8.

An aerial view of the San Francisco Campus vividly illustrates the extent to which the 1983 extension represents a singular moment in what had hitherto been a particularly homogenous campus, a rare anomaly that remains so to this day (Figure 2). To this date, the university had continued to grow, adding new faculties and extending both the original ones and the newer, but always following the spirit of the buildings designed by Borobio and Beltrán. This meant volumes resulting from the linear concatenation of parallelepipeds, symmetrical layouts, façades with a regular arrangement of openings, and the massive use of brick, which, combined in different proportions, ensured their integration into the surrounding built environment. Even other buildings that are seemingly discordant, such as the twin projects by the architects José Luis García-Marquina and Gloria Rahola for the faculties of Mathematics (197779) and Geology $(1978-85)^{9}$, with their facades clad in prefabricated concrete, ultimately follow the established layout patterns: a linear body with a symmetrical arrangement accessed via stairs in the centre of its long side in the first case, and an $L$ shape with sides of unequal length and height in the second. The same might be said of the Faculty of Education (2009-2013), the last of the major projects built to date on the campus, by Javier Maya and Estela Arteche (MAAR). Despite its broken geometries and its exposed concrete materiality, this new faculty is still a sculpture in the round whose volume articulates the space around it.

In contrast to all these, the building by Cisneros, Carmona, Fernández and Elorza made use of its location on the plot behind the first building of Borobio and Navarro and bordering calle Domingo Miral to introduce a mode of insertion and a formal vocabulary that set it apart from the other buildings and, therefore, from the very building they were extending. But at the same time, it responded "to a holistic approach" that sought to achieve "a complete and indivisible faculty as a whole"10 (figure 4). The trapezoidal plot on which the building was erected was a residual space, with the feel of a back yard, that had been occupied since the 1950s by several sports courts incongruously arranged on a diagonal. This space was limited on its two other sides by two smaller constructions. To the west, the reduced volume of the Institute of Education Sciences (1963-68), from a position almost tangent to the plane delimited by the lateral façade of the original building, constrained the plot and limited its expansion. To the east, somewhat further away, the Borobio and Beltrán teachers' residence had an even smaller footprint, but its 11-storey height gave it a notable presence.

Given this context, the architects proposed a solution that would create a building turned in on itself and that would blend into its surroundings. In the original project, the north-west façade was enveloped by a huge metal scaffolding, a latticework covered with vegetation, ultimately not built, which would have further concealed its presence. These transparent metallic structures formalised the transition between the new lecture room building and the wooded areas outside - the latter would only be executed in a much reduced version -, while the rest of the perimeter would be enclosed by a simpler metallic fence that gave the complex an unfinished appearance. The surrounding conditions meant that external access had to be from the east, where a stepped plaza spread along the side of the pre-existing building, taking in the pedestrian flow coming from the east. At its upper level, this plaza was inserted under the continuation of the short arm of the $U$ shaped by the old building, an area where the two volumes meet, marked by a double-height portico that echoed the language of the main façade of the original building. This piece acted as a buffer towards the internal garden while giving access to the building (figure 5).

On the other hand, the layout of the building replicated to a certain extent the U-shape of the original, but in this case starting from a single circular piece that enveloped the quasi-cubic aula magna of the first building -enclosing it in a garden only accessible from inside the building- and whose arms were extended to connect with it (figure 6). This asymmetrical crescent, with an arm span similar to that of the Borobio and Navarro building, would house a complex system of both vertical and horizontal circulation and socialisation spaces for students and teaching, research and administrative staff, a new and vital interior space, complex and rich in spatial relations. The connection with the original faculty was also achieved through this circulation space over its two floors, which ensured the correct 
functioning of the whole. Thus, the design combined autonomy and a distinct formal personality with both physical and functional subordination to the original building. This generates a piece whose volumetric rotundity is compatible with its user-friendliness, in such a way that the perceived scale belies the size of the constructed volume.

\section{CLUSTER FACULTY (CLUSTER VS. CLOISTER)}

"What we are after is something more complex... We are more concerned with 'flow' than with 'measure'. We have to create architecture [...] which, through built form, can make meaningful the change, the growth, the flow, the vitality of the community"11.

Around the said circulatory space, the different functions were plugged in, grouped in annulus sectors of varying depth and height that grew independently, extending as far as the plot limit allowed. Beginning in the east, next to the portico, a first volume housed the three large lecture halls, each seating 400 people, forming the largest block, and largely determining the perception of the faculty from the outside, by offering its side wall as a backdrop to the entrance plaza. Behind this, a lower section, with its glass roof sloping outwards, housed a cascading space, which generated leisure areas while physically and visually connecting the access floor with the semi-basement. Beyond it, two larger blocks that repeated its programme on the ground and first floors (figure 7). The first, smaller, included the toilets and a lecture room for 200 students per floor. The second contained a further three lecture rooms on each level. Separated

p.97 from the latter, a block of similar height, but divided internally into four levels, housed the departmental spaces and resolved the transition to the 1972 extension, whose fenestration it reproduced.

This floor arrangement by uses not only responds to a logic of a better exploitation of the plot, but also to functional criteria. Thus, the larger lecture rooms, intended for the first two heavily attended course years connected to the hall, next to the entrance, while the remainder, intended for later course years, were arranged in front, with both areas separated by a common room with leisure areas and toilets. At the back, the department block allows the teaching staff direct access to the lecture halls and the existing department areas. Below all of them, the semi-basement floor completed the programme with a large area dedicated to seminars and other non-teaching spaces, connecting, in the area under the entrance portico, with the bar of the original building and its attached sunken courtyard. Seen from the air, the building appeared as a flat-roofed crescent whose saw-toothed back had metamorphosed, adapting to the irregular contour, until it filled the space delimited by the plot.

The genesis, the soul, and ultimately, the success of the project, lie, in any case, in the inner ring (figure 7). This is set out in its report, which establishes as basic architectural objectives the "creation of a nucleus-core, around which the life of the Faculty will be focused", and of "an exterior space specific to the Faculty of Law: [the] Cloister"12. Thus, as opposed to the free layout of the enclosed spaces, articulated in an elementary way, by the simple juxtaposition of a series of bodies arranged radially, the inner ring is a complex and strict circulatory mechanism whose width is subjected to a series of dilations, subtractions and invasions. Among the first is the area adjacent to the entrance, where the circulating ring penetrates the body of the main classrooms to expand into a spacious vestibule. Among the subtractions, we can highlight the hollowing -complete on the ground floor and partial on the first floor, which overlooks the entrance- of the entrance portico, an operation that is repeated in the interior. Here, a vertical concentric void harbours the stairs, lying against the walls facing the courtyard, generating a whole series of crossed visions between levels. Despite its conceptual simplicity, the curved shape of the space produces a multiplication of perspectives that gives it a highly complex spatial sensation (figure 8).

The crucial moment in this space occurs, however, when it exceeds its strict function as an element of communication to become that social activator envisaged in the project report. As the authors state, the interior facade of this building opened towards the garden, acquires the form of a two-floor cloister: open to the elements, but covered, on its lower floor, exposed on the first floor. Both are joined to the interior levels of the original building. However, the height of the floor levels did not correspond to that of the inner circulation spaces defined above, but to the lower level of two flights of terraces, interior on the ground floor and open-air on the first floor, which by opening up the glazed walls, transformed the garden into an unexpected auditorium, a curia or, perhaps more appropriately, a courtroom.

This centripetal intention, to design a building turned inwards, would determine the choice of façade materials and the external image of the project. If the campus buildings are presented to the surroundings through uniform brick façades with a careful balance between wall and fenestration, the new building polarises this system into two antithetical responses: towards the cloister, a large part of the walls dissolves into large floor-to-ceiling glazed surfaces (Figure 9). From the outside, the building is broken up into a rosary of stone structures, built with a corrugated concrete block that responds to the context in a gentler way than might be expected. Its opaque envelopes, barely pierced in some areas by narrow, slit windows, construct a hermetic and abstract sculptural landscape which the observer's retina seems not to register. Only beneath the entrance level, when the surrounding terrain is manipulated in various ways, does the building boldly open up through large windows that look out onto its own secret garden (figure 10).

\section{p.99 A WORK BETWEEN TWO ERAS}

In their review of the architecture of the University of Zaragoza, Pilar Biel and Ascensión Hernández point out that "in the logical succession of architectural styles and trends produced over time, functionalism and brutalism would be followed by the emergence of postmodernism, which also has its manifestation in our university architecture [with a 
notable example in] the extension of the Faculty of Law"13. The first category would include some of the architecture p.100 designed in the 1960s, while the authors assign the buildings of García-Marquina and Rahola to the second, which certainly corresponds to the conventional use of the term. But the last assignment is more problematic. Perhaps it is the echoes of Greco-Roman architecture (figure 9) - "agora" or "classical theatre" are some of the terms used to describe the project- or a certain picturesque air conferred by the interior raised walkway, evoking other pergolas common to the era's post-modern language, which lead the authors to this categorisation. Perhaps it is the quotations imported from pre-existing buildings. Despite its relative formal autonomy, the building incorporates some nods in its encounter with them: the entrance portico is, in terms of proportion and geometry, a reworking of the one built by Borobio and Beltrán on the main façade (figure 1 and figure 5). And the same occurs in the new department block, where the lecture room building metamorphoses and creates a distorted doppelganger of the original, a half-hybrid of each that assists the transition between the old and the new. In any case, the irony or the metalinguistic intent that is supposed to be present in the postmodern game is missing. The two aforementioned elements are rather simply imports or borrowings that are introduced to create a series of visual rhymes. They are mimetic gestures rather than semantic games whose anamnestic capacity is, in this sense, very limited ${ }^{14}$.

Thus, despite the fact that the building fits chronologically into postmodernity, there is little of postmodernism in its design, that actually fits in with a distinctly modern tradition, both regarding its heroic phase and its interpretation from the late 1950s. It is inevitable, for example, that we should perceive echoes of Alvar Aalto both in the volumes and the floor plans, that remind us of designs such as the Wolfsburg Cultural Centre in Berlin (1959-1962). At the same time, radial forms, arranged circular fashion, whether complete or not, were common compositional schemes in modern architecture during the 1960s and subsequent years, being particularly present in the work of architects attached in one way or another to Brutalism. These circular forms are exemplified by John M. Johansen's US Embassy in Dublin (1959-64), or Kenzo Tange's Yukari Bunka kindergarten in Tokyo (1967), which played with the different lengths of the sectors to adapt to a site with a particularly irregular perimeter. It also shares strategies with contemporary interventions in university complexes such as Keble College Oxford where the De Breyne Building / Hayward Quad (1977) by Ahrends, Burton and Koralek defined the perimeter by means of a curvilinear piece that enveloped the original Victorian buildings. With a hermetic brick façade to the exterior, the building dematerialised into the interior garden by means of a continuous glass enclosure forming a covered, half-buried cloister on the lower floor. Not dissimilar was the older New Court (1966-1970) in Christ's College, by Denys Lasdun, whose now defunct - and slightly curved - original facade had a stepped volume that spilled into the garden space in the block's interior.

In Spain these circular arrangements may be found, particularly, in the work of Fernando Higueras, among others, throughout the 1960s. Particularly notable are the design for the competition for the Centro de Restauraciones Artísticas (Madrid, 1961), carried out jointly with Rafael Moneo, and the project finally built with Antonio Miró, now as the Centro Nacional de las Artes y de la Cultura de Madrid (1967-1970), both with a central circular courtyard as the p.101 heart and organising element of the project. Also relevant are his proposals for the competition for the new Opera House in Madrid (1963) or for the multi-purpose building in Monte Carlo (1969), in which the circular layout was developed radially in a centrifugal manner.

However, unlike these cases, the building does not embody that muscular architecture, characterised by a certain structural exhibitionism allied with a use of concrete that typifies the branch of late-modernism usually identified with Brutalism. Little of the apparent asceticism - and real sophistication - derived from the heroic use of materials is in evidence, but rather an understated pragmatism, which uses solutions of, a priori, little pedigree, and which, with the evident contrast, in the "honesty" of the material expression, brings this work closer to the work that Alison and Peter Smithson, notable brutalists of a different stamp, were realising at this time at the University of Bath.

In actual fact, the similarities with the Smithsons' work can be traced even further back. We can cite, for example, the overlap that occurs in the view from the entrance, where the side elevation of the large classroom volume raised on pillars, flanked by a ramp in with an "elevated street" behind, can quite reasonably be said to resemble, one of the British couple's most memorable images (figure 5) ) $^{15}$ : the well-known perspective drawing of their proposal for the extension of the University of Sheffield (1953), with its aerial street and the not very different volume of the lecture building in the foreground.

The same can be said of Bath's buildings, and particularly of its more prosaic architectural pieces, such as the unfinished Arts Barn (1980-90), with which this building is linked not only by the massive use of the concrete block, or the emphasis on the sloping lines introduced in the elevations by the roofs and exterior vertical communications, but also by a certain formal rawness derived from a proverbial matter-of-fact-ness in the resolution of functional problems. One of the a priori most surprising features of the lecture room building's aforementioned elevations is the presence of a small piece attached to its outer edge that lessens its formal roundness. Prosaically, it is the balcony parapet of an external emergency exit that not only becomes a particularly important feature of both adjacent elevations, but forms an unexpected social space in breaks between classes (figure 11).

This is precisely where the project most reflects back to the point of origin of the Smithsons' (new) Brutalism as a criticism of the deficiencies of modernity. As early as the Doorn Manifesto (1954), the revision of the modern movement promoted by Team $X$ had already emphasised the need to design meeting spaces that responded to different scales of gatherings and "the desire to produce [spaces] in which vital human associations would be expressed"16 at a time p.102 when "the social structure which the urban planner has to shape is not only different, but much more complex than it had 
been before"17. The meeting space, as the project report states, is one of the germinal elements of the design which in its cloister-garden, or nucleus-core ${ }^{18}$ (figure 12), found not only its own unique space on the campus, but also a place of a scale in keeping with the group of men and women, students, teachers and administrative staff it serves. And it did so on a campus essentially built under Franco's regime, a campus of polarised interior/exterior relations, with strictly delineated boundaries between the built structures and the vast outside common spaces.

The leitmotiv of the Faculty of Law extension was the creation of a community space in the sense of "community generator", and this is certainly something to which the elaborate polymorphic nature of the interior, multi-levelled circulation space at various level has clearly contributed. If the Smithsons' intention was for their elevated communication galleries to become "real street[s] in the air, [giving] access to enough people to become a social entity", 19 this aspect was essential in the new lecture room building whose inner ring left behind any spatial distinction between corridor, vertical communications and meeting space, all of them marked in the original project with the epigraph "classes

p.103 breaktime". As Michael Kubo, Chris Grimley and Mark Pasnik point out, Brutalism was "originally seen as reflecting the democratic attitudes of a powerful civic expression"20 whose material honesty, Nikil Saval adds, "was allied to the rough, prosaic goals of social democracy".

Brutalism is, as Michael J. Lewis has noted, "the vernacular expression of the welfare estate"21, and this is even truer of its original incarnation, before the term took on a stylistic connotation. Various authors have pointed to the socialist - or social democratic - roots associated with the New Brutalism programme 22 , and the democratisation of welfare implied by its quest to provide spaces that would activate social exchange, civic activity and, ultimately, participation in community life by men and women of all social strata. The desire of Cisneros, Carmona, Fernández and García Elorza to create a nucleus that would activate the life of the faculty and, consequently, spawn exchanges between all members of the education community and especially the students, certainly appears to point in this direction (figure 14). And it did so at a time when, for the first time in Spain, equal access to university was being consolidated, both in terms of social class and gender: the Smithsons' striking perspectives, as Reyner Banham noted in "The New Brutalism", validated "the presence of human beings as part of the total image, [with] photographs of people pasted on to the drawings, so that the human presence almost overwhelmed the architecture "23. Although not in photographic images, the nine cross sections contained in the University of Zaragoza Faculty of Law extension implementation project showed a sizable population of 180 human figures arranged to draw attention to the meeting spaces. However, if in their project proposal for the Golden Lane Estate (1953), Alison and Peter Smithson's perspectives prominently included Terence Conran, Jawaharlal Nehru or Gerard Philipe ${ }^{24}$, in this new lecture room building the sections appeared to be populated, finally, by an almost equal number of male and female students (figure 15).

\section{AN INVISIBLE BUILDING, AN INNER WORLD}

A testimony, perhaps, to the success of this search for an inner world achieved without fanfare is the fact that, almost forty years after its construction began, the 1983 building is, except for those who use it, scarcely noticed by the

p.104 public. With a discretion that belies both its bold volumetry and the large area it takes up, the new Law Faculty lecture theatre is a building that pedestrians only perceive as fragments, in oblique and fleeting glimpses captured as they move between other points on the campus and immediately forget; a building whose real magnitude can only be appreciated in its aerial view (figure 4). This is the result of both its position, squeezed in between other pre-existing or later constructed buildings, that have eliminated any possibility of a complete uninterrupted view, and the unhelpful treatment of its surroundings. Perhaps, in the end, this invisibility, both intended and fortuitous, has only helped guarantee the preservation of its ability to create an interior world at odds with the rest of the early buildings on the San Francisco Campus: a place that combines grand gestures with the everyday, which remains a time capsule both of the moment in which it was built and of the perhaps excessively optimistic ambitions of other periods of modernity.

1. The project would be the first to be promoted by the university itself as a result of the University Reform Law approved that year. GONZÁLEZ MARTíNEZ, Carlos; BIEL IBÁÑEZ, Pilar; HERNÁNDEZ MARTíNEZ, Ascensión, coords. La Universidad de Zaragoza: arquitectura y ciudad. Zaragoza: Universidad de Zaragoza, 2008, pp. 99-161.

2. In 1983, Aragon counted just 16 women architects enrolled in its Official Chamber, of whom Isabel Garcia Elorza had become the third member in 1971. The first woman architect to join Aragon's Chamber, in 1968, was Elvira Adiego, graduate of the School of Architecture of Madrid.

3. VÁZQUEZ ASTORGA, Mónica. José Borobio. Su aportación a la arquitectura moderna Zaragoza: Delegación del Gobierno en Aragón. Caja de Ahorros de la Inmaculada, 2007, pp. 228-234.

4. BIEL IBÁÑEZ, Maria Pilar. Autarquía y arquitectura industrial en Zaragoza. In: Artigrama, 2003, no. 18, pp. 527-548. DIÉGUEZ, Sofia. Arquitectura y town planning durante la autarquía. In: Antonio BONET CORREA (coord.), Arte del Franco regime. Madrid: Cátedra, 1981, pp. 47-76.

5. Indeed, the current Paraninfo of the University of Zaragoza, built by Ricardo Magdalena as the Hospital Clínico or Faculty of Medicine and Science between 1886 and 1893 , would undergo a substantial renovation by Borobio and Beltrán Navarro between 1957 and 1963.

6. In the academic year $1972-73$, almost 7000 Law students in the whole country were women, who represented almost $26 \%$ of enrolments. Three years later, this number had increased to 16,000 , being $34 \%$ of the total. Although the female pioneers of law studies in Spain can be traced back to the last quarter of the 19 th century, from the Second Republic until 1966, the legal professions - except for attorneys - would remain closed to women. See CAUSAPÉ GRACIA, Belén. La Facultad de Derecho de Zaragoza desde una perspectiva histórica de género. In: Josefa Dolores RUIZ RESA, ed. Las mujeres y las profesiones jurídicas. Madrid: Editorial Dykinson, 2020, pp. 23-41.

7. In reality, the project was even more ambitious, as a full refurbishment of the pre-existing building was envisaged that would allow both to function as a whole. However, in the end the university decided to abandon the idea. Later, between 1992 and 1994, the architect Basilio Tobías carried out the final expansion. The last more orthodox project 
would increase the surface area and height of Borobio y Beltrán's original building by adding an extra slightly recessed storey. The intervention would have two opposing effects: on the one hand, it reinforced the presence of the faculty in the central square which it overlooks, increasing its scale to make it more in keeping with it; and, on the other, it contributed to the imperceptibility of the new lecture theatre, a feature which, it is argued in this text, is, to a certain extent, one of its hallmarks. See GONZÁLEZ MARTíNEZ, C.; BIEL IBÁÑEZ, P.; HERNÁNDEZ MARTíNEZ, A., coords, op. cit. supra, nota 1

8. KING, Stephen. Secret window, secret garden. In: Four Past Midnight: Stories. New York: Pocket Books, 2017, p.406.

9. Although José Luis García-Marquina was officially the architect of the Faculty of Mathematics, in an interview conducted by the authors with Gloria Rahola on 13 April 2020 , she confirmed that she had co-authored the project. The Faculty of Geology is still officially the work of both architects.

10. CISNEROS LACRUZ, Saturnino; CARMONA MATEU, Juan Antonio; FERNÁNDEZ RAMíREZ, Manuel; GARCÍA ELORZA, Isabel. Project Report. In: Proyecto de ejecución, ampliación y reforma de la Facultad de Derecho (Zaragoza), 1983, p. 10.

11. SMITHSON, Alison; SMITHSON, Peter. Cluster City: A New Shape for the Community. In: The Architectural Review, 1957, vol. 122, n. ${ }^{\circ} 730$, pp. $333-336$.

12. CISNEROS LACRUZ, S.; CARMONA MATEU, J. A.; FERNÁNDEZ RAMÍREZ, M.; GARCÍA ELORZA, l., op. cit. supra, nota 13.

13. BIEL IBÁÑEZ, María Pilar; HERNÁNDEZ MARTíNEZ, Ascensión. Arquitectura y arquitectos de la Universidad de Zaragoza, op. cit. supra, nota 1, p. 280. Further on, the authors add: "The Law lecture hall by the architects Saturnino Cisneros Lacruz, Manuel Fernández Ramírez, Isabel García Elorza and Juan Antonio Mateu, stands out for its semi-spherical [sic] floor plan, like a classical theatre in which the closed character of its walls is contrasted with the open character of the agora around which the classrooms are open and which allows a 'playful' use of a teaching space".

14. ECO, Umberto. Postmodernism, irony, playfulness. In: Apostilles to The Name of the Rose and translation of the Latin texts. Barcelona: Círculo de Lectores, 1997, pp. 55-64. In this respect, and despite being somewhat anecdotal as a source of reference on postmodernism, we refer to the reflections to this effect made by Eco in this text, and especially to his comment that "in the same artist - a work, in this case - the modern and postmodern moments can coexist, or follow one another at a short distance, or alternate", which, if Biel's categorisation is accepted, would take on a literal meaning here.

15. In his seminal "The New Brutalism", Reyner Banham argues that one of its characteristics was "the need for the form to be apprehensible and memorable". BANHAM, Reyner. The New Brutalism. In: The Architectural Review, 1955, vol. 118, n. ${ }^{\circ} 708$, pp. 354-361.

16. HEUVEL, Dirk van der; RISSELADA Max, eds. Team 10: In Search of a Utopia of the Present 1953-1981. Rotterdam: NAi Publishers, 2005, p. 43. Original quote: "[...] their desire to produce towns in which vital human associations were expressed". BANHAM, R., op. cit. supra, nota 15.

17. SMITHSON, Alison; SMITHSON, Peter. An Alternative to the Garden City Idea. In: Architectural Design, July 1956, vol. 26, n. ${ }^{\circ}$ 7, pp. $229-231$.

18. It is symptomatic of the specific use of this terminology The references to "heart" and "nucleus" became common in modernity at least from CIAM 8: The Heart of the City, as evidence, for example TYRWHITT, Jaquelin. Cores within the Urban Constellation. In: Congrès Internationaux d'Architecture Moderne. The Heart of the City: towards the humanization of urban life. London: Lund Humphries, 1951.

19. SMITHSON, Alison; SMITHSON, Peter. The Charged Void: Architecture. New York: Monacelli Press, 2002. P. 86

20. KUBO, Michael; GRIMLEY, Chris; PASNIK, Mark. Brutal. In: Clog, February 2013, p. 166. In the text, the authors argue that "the reduction of Brutalism to a stylistic label exclusively associated with concrete coincides will a change of attitude towards the Government and the decline of state investment in the public domain. Originally seen as reflecting the democratic attitudes of a powerful civic expression (authenticity, honesty, openness and strength), the forceful nature of the Brutalist aesthetic eventually came to signify precisely the opposite: hostility, coldness, inhumanity".

21. SAVAL, Nikil. Brutalism Is Back. In: The New York Times Magazine, 2016, n. ${ }^{\circ} 23$, p. 53.

22. BULLOCK, Nicolas. Building the Socialist Dream or Housing the Socialist State? Design versus the Production of Housing in the 1960s. In: Mark CRISON; Claire ZIMMERMAN, eds. Neo-Avant Garde and Postmodern: Post-war Architecture in Britain and Beyond. New Haven: Yale University Press, 2010, pp. $321-342$.

23. SMITHSON, A.; SMITHSON, P., op. cit. supra, nota 17.

24. CAPDEVILA CASTELLANOS, Iván; IBORRA PALLARÉS, Vicente. Drawing a New Architectural Paradigm. In: Carlos L. MARCOS, ed. Graphic Imprints: The Influence of Representation and Ideation Tools in Architecture. New York: Springer, 2019, pp. 274-284. DOI: 10.1007/978-3-319-93749-6_23 

CITY. Darío Álvarez Álvarez • LINAZASORO EN REIMS. EL ESPACIO PÚBLICO COMO MEMORIA DEL LUGAR / LINAZASORO IN REIMS. THE PUBLIC SPACE AS THE MEMORY OF THE PLACE. Victoriano Sainz Gutiérrez • EDITAR VS. CONSTRUIR: UNA ECOLOGÍA DE LO INVISIBLE. AMPLIFICAR LA COMPRENSIÓN DE LAS TÉCNICAS DE PROYECTO / EDITING VS. BUILDING: AN ECOLOGY OF THE INVISIBLE. AMPLIFYING THE UNDERSTANDING OF ARCHITECTURAL DESIGN TECHNIQUES. Paula Victoria Álvarez Benítez • CUANDO LA PINTURA AMPLÍA LA ARQUITECTURA: INTERVENCIONES REALIZADAS EN EL ESPACIO PÚBLICO / WHEN PAINTING ENHANCES ARCHITECTURE: INTERVENTIONS IN A PUBLIC SETTING. Aurora Alcaide-Ramírez; Ana Ruiz-Abellón • UN EDIFICIO INVISIBLE. NUEVO AULARIO DE LA FACULTAD DE DERECHO DE LA UNIVERSIDAD DE ZARAGOZA (1983-1996) / AN INVISIBLE BUILDING. NEW LECTURE ROOM BUILDING OF THE FACULTY OF LAW OF THE UNIVERSITY OF ZARAGOZA (1983-1996). Luis Miguel Lus-Arana; Lucía Carmen Pérez-Moreno • ARQUITECTURAS AMPLIADAS. EL PABELLÓN DE EXPOSICIONES EN LA CASA DE CAMPO DE MADRID) / EXPANDED ARCHITECTURES. THE EXHIBITION PAVILION AT THE CASA DE CAMPO IN MADRID. José de Coca Leicher $\bullet$

- RAFAEL MONEO VALLÉS: LA VIDA DE LOS EDIFICIOS. LA MEZQUITA DE CÓRDOBA, LA LONJA DE SEVILLA Y UN CARMEN EN GRANADA . Víctor Pérez Escolano • FRANCISCO DE GRACIA: CONSTRUIR EN LO CONSTRUIDO. LA ARQUITECTURA COMO MODIFICACIÓN. Pablo Diañez Rubio • FRÉDÉRIC DRUOT, ANNE LACATON \& JEAN-PHILIPPE VASSAL PLUS: LA VIVIENDA COLECTIVA. TERRITORIO DE EXCEPCIÓN. Javier Terrados Cepeda.
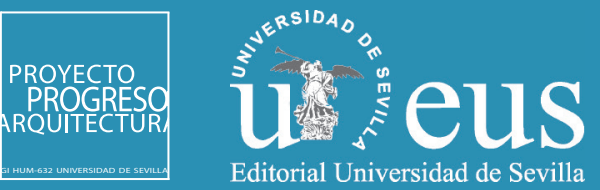